\title{
COUNTABLY GENERATED DOUGLAS ALGEBRAS
}

\author{
KEIJI IZUCHI
}

\begin{abstract}
Under a certain assumption of $f$ and $g$ in $L^{\infty}$ which is considered by Sarason, a strong separation theorem is proved. This is available to study a Douglas algebra $\left[H^{\infty}, f\right]$ generated by $H^{\infty}$ and $f$. It is proved that (1) ball $\left(B / H^{\infty}+C\right)$ does not have exposed points for every Douglas algebra $B,(2)$ Sarason's three functions problem is solved affirmatively, (3) some characterization of $f$ for which $\left[H^{\infty}, f\right]$ is singly generated, and (4) the $M$-ideal conjecture for Douglas algebras is not true.
\end{abstract}

Let $H^{\infty}$ be the space of bounded analytic functions on the unit disk. A uniformly closed subalgebra between $H^{\infty}$ and $L^{\infty}$ is called a Douglas algebra. By ChangMarshall's theorem $[\mathbf{3}, \mathbf{1 9}]$, a Douglas algebra is generated by $H^{\infty}$ and complex conjugates of some inner functions. A Douglas algebra is called singly (countably, respectively) generated if it is generated by $H^{\infty}$ and a complex conjugate of only one (countably many) inner function(s). In this paper, we investigate a Douglas algebra $\left[H^{\infty}, f\right]$ which is generated by $H^{\infty}$ and $f$ in $L^{\infty}$. By Chang-Marshall's theorem, it is easy to see that $\left[H^{\infty}, f\right]$ is countably generated. To study $\left[H^{\infty}, f\right]$, we have to study the behavior of $f$ on $M\left(L^{\infty}\right)$. Let $N(f)$ equal the closure of

$$
\bigcup\left\{\operatorname{supp} \mu_{x} ; x \in M\left(H^{\infty}+C\right) \text { and } f\left|\operatorname{supp} \mu_{x} \notin H^{\infty}\right| \operatorname{supp} \mu_{x}\right\} ;
$$

roughly speaking $N(f)$ is a subset of $M\left(L^{\infty}\right)$ on which $f$ is not analytic. Properties of $N(f)$ play important roles in studying Douglas algebras. $N(f)$, especially $N(\bar{I})$ where $I$ is an inner function, is studied in $[\mathbf{1 3}]$.

The key theorem (Theorem 2.1 given in $\S 2$ ) is that if either $f \mid \operatorname{supp} \mu_{x}$ or $g \mid \operatorname{supp} \mu_{x}$ belongs to $H^{\infty} \mid \operatorname{supp} \mu_{x}$ for every $x \in M\left(H^{\infty}+C\right)$ then $N(f) \cap N(g)=\varnothing$. When $f$ and $g$ are inner functions, this fact is already proved in [13]. The above assumption is considered by Sarason [22], and he showed that either $f \mid Q$ or $g \mid Q$ belongs to $H^{\infty} \mid Q$ for every $Q C$-level set $Q$ under the above assumption. Our theorem with Corollary 2.1 gives more striking separation than Sarason's. Using our separation theorem, we study singly or countably generated Douglas algebras. In [14], the author showed that a Douglas algebra $B$ is singly generated if and only if ball $\left(B / H^{\infty}+C\right)$ has extreme points. In $\S 3$, we shall give also a geometrical characterization of countably generated Douglas algebras. And we shall show that ball $\left(B / H^{\infty}+C\right)$ does not have exposed points for every Douglas algebra $B$. In $[\mathbf{2 2}$, p. 471], Sarason proposed a problem that the above mentioned Sarason theorem is still true for three functions. In $\S 4$, we shall give an affirmative answer. In $\S 5$, we study a special sequence of $Q C$-level sets which will be called strongly discrete. Using a property of such a sequence, given in Theorem 5.1, we shall prove

Received by the editors January 11, 1986.

1980 Mathematics Subject Classification (1985 Revision). Primary 46J15, 46J30; Secondary $30 \mathrm{H} 15,46 \mathrm{~B} 20$. 
a theorem which is more precise than Gorkin's given in [8, Theorem 2.1]. In $\S 6$, we shall give equivalent conditions on $f$ for which $\left[H^{\infty}, f\right]$ is singly generated. This answers Marshall's problem given in $[\mathbf{1 9}]$. In $\S 7$, we shall give a negative answer of the $M$-ideal conjecture [18].

1. Preliminaries. Let $A$ be a uniformly closed subalgebra of $C(K)$, the space of continuous functions on a compact Hausdorff space $K$. We denote by $M(A)$ the maximal ideal space of $A$ equipped with the weak*-topology and by $\partial A$ the Shilov boundary for $A$. For $f \in C(\partial A),\|f\|$ means the supremum norm of $f$ and $\bar{f}$ means the complex conjugate of $f$. A closed subset $E$ of $\partial A$ is called a peak set for $A$ if there is a function $f$ in $A$, which is called a peaking function for $E$, such that $\|f\|=1$ and $E=\{x \in \partial A ;|f(x)|=1\}=\{x \in \partial A ; f(x)=1\}$. If $E$ is an intersection of peak sets, it is called a weak peak set for $A$. A measure $\mu$ on $\partial A$ is called an annihilating measure for $A, \mu \perp A$, if $\int_{\partial A} f d \mu=0$ for every $f \in A$. Gamelin's book [4] is a good reference for uniform algebras.

Let $D$ be the open unit disk. Let $L^{\infty}$ be the space of bounded measurable functions on $\partial D$ with respect to the normalized Lebesgue measure $d \theta / 2 \pi$. We identify a function $f$ in $H^{\infty}$ with its boundary function. Then $H^{\infty}$ is an essentially uniformly closed subalgebra of $L^{\infty} . H^{\infty}+C$ is the smallest Douglas algebra which contains $H^{\infty}$ properly $[\mathbf{2 1}]$, where $C$ is the space of continuous functions on $\partial D$. We put $X=M\left(L^{\infty}\right)$, then $X$ may be identified with $\partial H^{\infty}$. We note that $M\left(H^{\infty}+C\right)=$ $M\left(H^{\infty}\right) \backslash D$, and $D$ is weak ${ }^{*}$-dense in $M\left(H^{\infty}\right)$ by the corona theorem (see $\left.[6]\right)$. For a subset $E$ of $M\left(H^{\infty}\right)$, we denote by $\mathrm{cl} E$ the weak*-closure of $E$ in $M\left(H^{\infty}\right)$. For a point $x$ in $M\left(H^{\infty}\right)$, we denote by $\mu_{x}$ the unique representing measure on $X$ for $x$, and by $\operatorname{supp} \mu_{x}$ the closed support set for $\mu_{x}$. Supp $\mu_{x}$ is a weak peak set for $H^{\infty}\left[10\right.$, p. 207], and it is easy to see that $H^{\infty} \mid \operatorname{supp} \mu_{x}$ does not contain any nonconstant real functions. By Sarason [20],

$$
H^{\infty}+C=\left\{f \in L^{\infty} ; f\left|\operatorname{supp} \mu_{x} \in H^{\infty}\right| \operatorname{supp} \mu_{x} \text { for every } x \in M\left(H^{\infty}+C\right)\right\} .
$$

We use the notation $m$ for the representing measure for the point 0 in $D$, that is, $\int_{X} f d m=\int_{\partial D} f d \theta / 2 \pi$ for every $f \in H^{\infty}$. For $f \in L^{\infty}$ and a Douglas algebra $B$, we put $\|f+B\|=\inf \{\|f+h\| ; h \in B\}$, the quotient norm of $L^{\infty} / B$. For a subset $E$ of $L^{\infty}$, we denote by $[E]$ the uniformly closed subalgebra generated by $E$.

Put $Q C=\left(H^{\infty}+C\right) \cap \overline{\left(H^{\infty}+C\right)}$ and $Q A=H^{\infty} \cap Q C$. By [20],

$$
Q C=\left\{f \in L^{\infty} ; f \mid \operatorname{supp} \mu_{x} \text { is constant for every } x \in M\left(H^{\infty}+C\right)\right\} .
$$

Then $Q C$ is a $C^{*}$-subalgebra of $L^{\infty}$. Hence there is a continuous onto map $\pi: X \rightarrow$ $M(Q C) ; f(\pi(x))=f(x)$ for every $f \in Q C$. A closed subset $\pi^{-1}(y), y \in M(Q C)$, is called a $Q C$-level set. A $Q C$-level set is a weak peak set for $Q A$. For $x \in$ $M\left(H^{\infty}+C\right)$, there is a unique $Q C$-level set $Q_{x}$ such that $Q_{x} \supset \operatorname{supp} \mu_{x}$. We denote by $m_{0}$ the probability measure on $M(Q C)$ such that $\int_{M(Q C)} f d m_{0}=\int_{\partial D} f d \theta / 2 \pi$ for every $f \in Q A$. Then we have $m\left(\pi^{-1}(E)\right)=m_{0}(E)$ for measurable subsets $E$ of $M(Q C)$.

For $f \in L^{\infty}$, we put

$$
N(f)=\text { the closure of } \bigcup\left\{\operatorname{supp} \mu_{x} ; f\left|\operatorname{supp} \mu_{x} \notin H^{\infty}\right| \operatorname{supp} \mu_{x}\right\} \text {, }
$$

and

$$
Q(f)=\bigcup\left\{\pi^{-1}(y) ; f\left|\pi^{-1}(y) \notin H^{\infty}\right| \pi^{-1}(y), y \in M(Q C)\right\} .
$$

Generally $Q(f)$ is not a closed subset of $X$. 
A Blaschke product with zeros $\left\{z_{n}\right\}_{n=1}^{\infty}$ in $D$ is a function of the form

$$
b(z)=\prod_{n=1}^{\infty} \frac{-\bar{z}_{n}}{\left|z_{n}\right|} \frac{z-z_{n}}{1-\bar{z}_{n} z}
$$

for $z \in D$, where $\sum_{n=1}^{\infty} 1-\left|z_{n}\right|<\infty$. If $\left\{z_{n}\right\}_{n=1}^{\infty}$ satisfies moreover

$$
\inf _{n} \prod_{m \neq n}\left|\frac{z_{n}-z_{m}}{1-\bar{z}_{m} z_{n}}\right|>0 \quad\left(\lim _{n \rightarrow \infty} \prod_{m \neq n}\left|\frac{z_{n}-z_{m}}{1-\bar{z}_{m} z_{n}}\right|=1 \text { respectively }\right),
$$

then $\left\{z_{n}\right\}_{n=1}^{\infty}$ is called interpolating (sparse), and $b(z)$ is called an interpolating (sparse) Blaschke product. These Blaschke products are inner functions, where a function $I \in H^{\infty}$ with $|I|=1$ on $X$ is called inner. If $I$ is an inner function, put $Z(I)=\left\{x \in M\left(H^{\infty}+C\right) ; I(x)=0\right\}$. Then $N(\bar{I})=Q(\bar{I})=\bigcup\left\{Q_{x} ; x \in Z(I)\right\}$ [13, Theorem 1]. If $b$ is an interpolating Blaschke product with zeros $\left\{z_{n}\right\}_{n=1}^{\infty}$, then $\operatorname{cl}\left\{z_{n}\right\}_{n=1}^{\infty}$ is homeomorphic to the Stone-Cech compactification of $\left\{z_{n}\right\}_{n=1}^{\infty}$ and $Z(b)=\operatorname{cl}\left\{z_{n}\right\}_{n=1}^{\infty} \backslash\left\{z_{n}\right\}_{n=1}^{\infty}[10$, p. 205].

Let $Y$ be a Banach space. We denote by ball $Y$ the closed unit ball of $Y$. A point $y$ in ball $Y$ is called extreme if $\|y \pm x\| \leq 1, x \in Y$, implies $x=0$. A point $y$ in ball $Y$ is called exposed if there is a bounded linear functional $\psi$ of $Y$ such that $\|\psi\|=1, \psi(y)=1$ and $\psi(x) \neq 1$ for every $x \in$ ball $Y$ with $x \neq y$. We note that an exposed point is extreme. A closed subspace $Z$ of $Y$ is called an $M$-ideal of $Y$ if there is a projection $P$ from $Y^{*}$, the dual space of $Y$, onto the annihilating subspace of $Z$ in $Y^{*},\left\{f \in Y^{*} ; f=0\right.$ on $\left.Z\right\}$, such that $\|x\|=\|P x\|+\|x-P x\|$ for every $x$ in $Y^{*}$.

2. The main theorem. In this section, we shall show the following theorem and give its applications.

THEOREM 2.1. Let $f$ and $g$ be functions in $L^{\infty}$. If for every $x \in M\left(H^{\infty}+C\right)$ either $f\left|\operatorname{supp} \mu_{x} \in H^{\infty}\right| \operatorname{supp} \mu_{x}$ or $g\left|\operatorname{supp} \mu_{x} \in H^{\infty}\right| \operatorname{supp} \mu_{x}$, then $N(f) \cap N(g)=$ $\varnothing$.

To show Theorem 2.1, we need some lemmas.

LEMMA $2.1[\mathbf{2 4}]$. For an inner function $I$, there is an interpolating Blaschke product b such that $\left[H^{\infty}, \bar{b}\right]=\left[H^{\infty}, \bar{I}\right]$.

LEMMA 2.2. Let $B$ be a Douglas algebra. Then the following assertions are equivalent.

(i) There is a function $f$ in $L^{\infty}$ with $B=\left[H^{\infty}, f\right]$.

(ii) There is a sequence of interpolating Blaschke products $\left\{I_{n}\right\}_{n=1}^{\infty}$ with $B=$ $\left[H^{\infty},\left\{\bar{I}_{n}\right\}_{n=1}^{\infty}\right]$.

Proof. Let $f \in L^{\infty}$ with $B=\left[H^{\infty}, f\right]$. By Chang-Marshall's theorem, there is a sequence of inner functions $\left\{I_{n}\right\}_{n=1}^{\infty}$ such that $\bar{I}_{n} \in\left[H^{\infty}, f\right]$ and $\left\|I_{n} f+H^{\infty}\right\| \rightarrow 0$ $(n \rightarrow \infty)$. Then $\left[H^{\infty}, f\right] \subset\left[H^{\infty},\left\{\bar{I}_{n}\right\}_{n=1}^{\infty}\right] \subset\left[H^{\infty}, f\right]$, so $\left[H^{\infty}, f\right]=\left[H^{\infty},\left\{\bar{I}_{n}\right\}_{n=1}^{\infty}\right]$. By Lemma 2.1, we may take $I_{n}$ as an interpolating Blaschke product. Conversely suppose that $B=\left[H^{\infty},\left\{\bar{I}_{n}\right\}_{n=1}^{\infty}\right]$ for a sequence of inner functions $\left\{I_{n}\right\}$. We put $f=\sum_{n=1}^{\infty}\left|I_{n}+1\right| / 3^{n}$. If $I_{n} \mid \operatorname{supp} \mu_{x}, x \in M\left(H^{\infty}\right)$, is not constant, then 
$I_{n}\left(\operatorname{supp} \mu_{x}\right)=\partial D$. Hence $f \mid \operatorname{supp} \mu_{x}$ is constant if and only if $I_{n} \mid \operatorname{supp} \mu_{x}$ is constant for every $n$. Since real functions in $H^{\infty} \mid \operatorname{supp} \mu_{x}$ are constant functions for each $x \in M\left(H^{\infty}\right), M\left(\left[H^{\infty}, f\right]\right)=M\left(\left[H^{\infty},\left\{\bar{I}_{n}\right\}_{n=1}^{\infty}\right]\right)$. By Chang-Marshall's theorem, $f$ is the desired function.

The following lemma is a special case of Theorem 2.1 proved in [13, Corollary $3]$.

LEMMA 2.3. Let $I$ and $J$ be inner functions. If for every point $x$ in $M\left(H^{\infty}+C\right)$ either $\bar{I}\left|\operatorname{supp} \mu_{x} \in H^{\infty}\right| \operatorname{supp} \mu_{x}$ or $\bar{J}\left|\operatorname{supp} \mu_{x} \in H^{\infty}\right| \operatorname{supp} \mu_{x}$, then $N(\bar{I}) \cap N(\bar{J})=$ $\varnothing$.

LEMMA 2.4. Let $I$ be an interpolating Blaschke product. Let $E$ be a closed subset of $D$ such that $\operatorname{cl} E \backslash E \subset\left\{x \in M\left(H^{\infty}+C\right) ;|I(x)|=1\right\}$. Then for each $\varepsilon$ with $0<\varepsilon<1$, there is an interpolating Blaschke product $b$ satisfying that $I \bar{b}$ is $a$ finite Blaschke product and $|b| \geq \varepsilon$ on $E$.

ProOF. Let $\left\{z_{n}\right\}_{n=1}^{\infty}$ be the zero sequence of $I$. We denote by $I_{k}$ the interpolating Blaschke product with zeros $\left\{z_{n}\right\}_{n=k}^{\infty}$. By our assumption, there exists a constant $r$ such that $0<r<1$ and $|I| \geq \varepsilon$ on $\{z \in E ;|z|>r\}$. Since $\left|I_{k}\right| \rightarrow 1$ $(k \rightarrow \infty)$ uniformly on each compact subset of $D,\left|I_{k}\right| \geq \varepsilon$ on $\{z \in E ;|z| \leq r\}$ for sufficiently large $k$. Put $b=I_{k}$, then $b$ satisfies our assertion.

The following is a key lemma to prove Theorem 2.1 .

LEMMA 2.5. Let $\left\{I_{n}\right\}_{n=1}^{\infty}$ be a sequence of interpolating Blaschke products such that $\prod_{n=1}^{\infty} I_{n}$ is a Blaschke product. Let $g$ be a function in $L^{\infty}$. Suppose that for every $x \in M\left(H^{\infty}+C\right)$ either $g\left|\operatorname{supp} \mu_{x} \in H^{\infty}\right| \operatorname{supp} \mu_{x}$ or $\bar{I}_{n}\left|\operatorname{supp} \mu_{x} \in H^{\infty}\right| \operatorname{supp} \mu_{x}$ for all $n$. Then there exists a Blaschke product I such that

(i) $\left(\prod_{n=1}^{\infty} I_{n}\right) \bar{I} \in H^{\infty}$; consequently $N(\bar{I}) \subset N\left(\overline{\prod_{n=1}^{\infty} I_{n}}\right)$;

(ii) either $\bar{I}\left|\operatorname{supp} \mu_{x} \in H^{\infty}\right| \operatorname{supp} \mu_{x}$ or $g\left|\operatorname{supp} \mu_{x} \in H^{\infty}\right| \operatorname{supp} \mu_{x}$ for every $x \in$ $M\left(H^{\infty}+C\right) ;$ and

(iii) $N\left(\bar{I}_{n}\right) \subset N(\bar{I})$ for all $n$.

PROOF. By Lemma 2.2, there is a sequence of interpolating Blaschke products $\left\{J_{m}\right\}_{m=1}^{\infty}$ such that

$$
\left[H^{\infty}, g\right]=\left[H^{\infty},\left\{\bar{J}_{m}\right\}_{m=1}^{\infty}\right]
$$

By our assumption, for every $x \in M\left(H^{\infty}+C\right)$, either $\bar{I}_{n}\left|\operatorname{supp} \mu_{x} \in H^{\infty}\right| \operatorname{supp} \mu_{x}$ for all $n$ or $\bar{J}_{m}\left|\operatorname{supp} \mu_{x} \in H^{\infty}\right| \operatorname{supp} \mu_{x}$ for all $m$. By Lemma 2.3,

$$
N\left(\bar{I}_{n}\right) \cap N\left(\bar{J}_{m}\right)=\varnothing \quad \text { for every } n \text { and } m \text {. }
$$

Let $\left\{z_{n, k}\right\}_{k=1}^{\infty}$ be the zero sequence of $I_{n}$. Put $I_{0}=\prod_{n=1}^{\infty} I_{n}$. Since $I_{0}$ is a Blaschke product, we have

$$
\sum_{n=1}^{\infty} \sum_{k=1}^{\infty}\left(1-\left|z_{n, k}\right|\right)<\infty .
$$

For each $m$, we put

$$
U_{m, i}=\left\{z \in D ;\left|J_{m}(z)\right| \leq 1-1 / i,|z| \geq 1-1 / i\right\}
$$

for $i=1,2, \ldots$ Then $U_{m, i}$ is a closed subset of $D$. By (2) and (4), $I_{n}(=I)$ and $U_{m, i}(=E)$ satisfy the assumptions of Lemma 2.4. Because, if there is $x$ 
in $\operatorname{cl} U_{m, i} \backslash U_{m, i}$ with $\left|I_{n}(x)\right| \neq 1$, then $\left|J_{m}(x)\right| \leq 1-1 / i$, so we get $\operatorname{supp} \mu_{x} \subset$ $N\left(\bar{I}_{n}\right) \cap N\left(\bar{J}_{m}\right)$.

First we shall work on $J_{1}$, and we shall find a sequence of interpolating Blaschke products $\left\{b_{1, n}\right\}_{n=1}^{\infty}$ satisfying the following two conditions by induction.

(5) $I_{n} \bar{b}_{1, n}$ is a finite Blaschke product, and

(6) $\inf \left\{\left|\left(b_{1, i} b_{1, i+1} \cdots b_{1, n}\right)(z)\right| ; z \in U_{1, i}\right\}>1-1 / i$ for $1 \leq i \leq n$.

Applying Lemma 2.4 for $I_{1}$ and $U_{1,1}$, there is an interpolating Blaschke product $b_{1,1}$ such that $I_{1} \bar{b}_{1,1}$ is a finite Blaschke product and $\inf \left\{\left|b_{1,1}(z)\right| ; z \in U_{1,1}\right\}>0$. Suppose that $\left\{b_{1,1}, b_{1,2}, \ldots, b_{1, N}\right\}$ satisfies (5) and (6) for $1 \leq i \leq n \leq N$. For $1 \leq i \leq N$, we put

$$
c(N, i)=\inf \left\{\left|\left(b_{1, i} b_{1, i+1} \cdots b_{1, N}\right)(z)\right| ; z \in U_{1, i}\right\} .
$$

By $(6), c(N, i)>1-1 / i$. Also we put

$$
E=\bigcup\left\{U_{1, i} ; 1 \leq i \leq N+1\right\}
$$

then $I=I_{N+1}$ and $E$ satisfy the assumptions of Lemma 2.4. Let $\varepsilon$ be a constant satisfying

$$
1>\varepsilon>\max \left\{1-\frac{1}{N+1}, \frac{1-1 / i}{c(N, i)} ; 1 \leq i \leq N\right\} .
$$

By Lemma 2.4, there is an interpolating Blaschke product $b_{1, N+1}$ such that $I_{N+1} \bar{b}_{1, N+1}$ is a finite Blaschke product and

$$
\left|b_{1, N+1}\right| \geq \varepsilon \text { on } E \text {. }
$$

Thus we get the following inequalities.

For $1 \leq i<N+1$;

$$
\begin{aligned}
\inf \{ & \left.\left|b_{1, i} b_{1, i+1} \cdots b_{1, N+1}(z)\right| ; z \in U_{1, i}\right\} \\
& \geq \inf \left\{\left|b_{1, i} b_{1, i+1} \cdots b_{1, N}(z)\right| ; z \in U_{1, i}\right\} \inf \left\{\left|b_{1, N+1}(z)\right| ; z \in U_{1, i}\right\} \\
& >c(N, i) \varepsilon \text { by }(7),(8) \text { and }(10) \\
& >1-1 / i \text { by (9). }
\end{aligned}
$$

For $i=N+1$;

$$
\inf \left\{\left|b_{1, N+1}(z)\right| ; z \in U_{1, N+1}\right\} \geq \varepsilon>1-1 / N+1 \text { by (8), (9) and (10). }
$$

Consequently $\left\{b_{1,1}, b_{1,2}, \ldots, b_{1, N+1}\right\}$ satisfies (5) and (6). This completes the construction of $\left\{b_{1, n}\right\}_{n=1}^{\infty}$.

In the above proof, we use only the fact $N\left(\bar{J}_{1}\right) \cap N\left(\bar{I}_{n}\right)=\varnothing$ for $n=1,2, \ldots$ By (2) and (5), we have $N\left(\bar{J}_{2}\right) \cap N\left(\bar{b}_{1, n}\right)=\varnothing$. So we can repeat the above argument for $J_{2}$ and $\left\{b_{1, n}\right\}_{n=2}^{\infty}$, we remark that $n$ starts from 2. Then there is a sequence of interpolating Blaschke products $\left\{b_{2, n}\right\}_{n=2}^{\infty}$ such that $b_{1, n} \bar{b}_{2, n}$ is a finite Blaschke product for $n \geq 2$ and

$$
\inf \left\{\left|b_{2, i} b_{2, i+1} \cdots b_{2, n}(z)\right| ; z \in U_{2, i}\right\}>1-1 / i \text { for } 2 \leq i \leq n .
$$

Repeating the above argument several times, for each $m$ there is a sequence of interpolating Blaschke products $\left\{b_{m, n}\right\}_{n=m}^{\infty}$ such that

$$
b_{m, n} \bar{b}_{m+1, n} \text { is a finite Blaschke product for } m+1 \leq n,
$$


and

$$
\inf \left\{\left|b_{m, i} b_{m, i+1} \cdots b_{m, n}(z)\right| ; z \in U_{m, i}\right\}>1-1 / i \text { for } m \leq i \leq n .
$$

We put $I=\prod_{n=1}^{\infty} b_{n, n}$. By (3) and.(11), $I$ is a Blaschke product and $I_{0} \bar{I} \in H^{\infty}$, so we get (i). We shall prove that I satisfies (ii) and (iii).

To prove (ii), let $x \in M\left(H^{\infty}+C\right)$ with $g\left|\operatorname{supp} \mu_{x} \notin H^{\infty}\right| \operatorname{supp} \mu_{x}$. We shall prove $\bar{I}\left|\operatorname{supp} \mu_{x} \in H^{\infty}\right| \operatorname{supp} \mu_{x}$. By (1), there is an integer $m$ such that $\bar{J}_{m} \mid \operatorname{supp} \mu_{x} \notin$ $H^{\infty} \mid \operatorname{supp} \mu_{x}$, that is, $\left|J_{m}(x)\right|<1$. Take a positive integer $i_{0}$ with $m \leq i_{0}$ and

$$
\left|J_{m}(x)\right|<1-1 / i_{0} \text {. }
$$

Let $i \geq i_{0}$. By (4), (13) and the corona theorem, $x \in \operatorname{cl} U_{m, i_{0}} \backslash U_{m, i_{0}}$. Since $\left|b_{m, n}\right| \leq\left|b_{n, n}\right|$ on $D$ for $m \leq n$ by $(11)$, we have

$$
\begin{aligned}
\inf \left\{\left|\prod_{n=i}^{\infty} b_{n, n}(z)\right| ; z \in U_{m, i}\right\} & \geq \inf \left\{\left|\prod_{n=i}^{\infty} b_{m, n}(z)\right| ; z \in U_{m, i}\right\} \\
& \geq 1-1 / i \text { by }(12) .
\end{aligned}
$$

Then

$$
\left|\prod_{n=i}^{\infty} b_{n, n}\right| \geq 1-\frac{1}{i} \quad \text { on } \mathrm{cl} U_{m, i} \backslash U_{m, i} .
$$

By (5) and (11), $\left|I_{n}\right|=\left|b_{n, n}\right|$ on $M\left(H^{\infty}+C\right)$ for $n=1,2, \ldots$ By (2) and (4), $\left|I_{n}\right|=1$ on $\operatorname{cl} U_{m, i} \backslash U_{m, i}$ for $n=1,2, \ldots$ Thus

$$
|I|=\left|\prod_{n=1}^{\infty} b_{n, n}\right| \geq 1-\frac{1}{i} \quad \text { on } \operatorname{cl} U_{m, i} \backslash U_{m, i} .
$$

Since $\operatorname{cl} U_{m, i} \backslash U_{m, i} \subset \operatorname{cl} U_{m, j} \backslash U_{m, j}$ for $i \leq j$ by (4), we get

$$
|I| \geq 1-1 / i \text { on } \operatorname{cl} U_{m, i_{0}} \backslash U_{m, i_{0}} \quad \text { for every } i \geq i_{0} \text {. }
$$

Thus $|I|=1$ on $\operatorname{cl} U_{m, i_{0}} \backslash U_{m, i_{0}}$. Since $x \in \operatorname{cl} U_{m, i_{0}} \backslash U_{m, i_{0}},|I(x)|=1$. Hence $I$ is constant on $\operatorname{supp} \mu_{x}$, and $\bar{I}\left|\operatorname{supp} \mu_{x} \in H^{\infty}\right| \operatorname{supp} \mu_{x}$. This completes the proof of (ii).

Since $\left|I_{n}\right|=\left|b_{n, n}\right|$ on $M\left(H^{\infty}+C\right)$ for each $n$,

$$
|I|=\left|\prod_{n=1}^{\infty} b_{n, n}\right| \leq\left|b_{n, n}\right|=\left|I_{n}\right| \quad \text { on } M\left(H^{\infty}+C\right) .
$$

Thus we get $N(\bar{I}) \supset N\left(\bar{I}_{n}\right)$. This completes the proof.

PROOF OF THEOREM 2.1. Let $f$ and $g$ be functions in $L^{\infty}$ such that for every $x \in M\left(H^{\infty}+C\right)$ either $f\left|\operatorname{supp} \mu_{x} \in H^{\infty}\right| \operatorname{supp} \mu_{x}$ or $g\left|\operatorname{supp} \mu_{x} \in H^{\infty}\right| \operatorname{supp} \mu_{x}$. We shall show the existence of a Blaschke product $I$ such that

(a) either $\bar{I}\left|\operatorname{supp} \mu_{x} \in H^{\infty}\right| \operatorname{supp} \mu_{x}$ or $g\left|\operatorname{supp} \mu_{x} \in H^{\infty}\right| \operatorname{supp} \mu_{x}$ for every $x \in$ $M\left(H^{\infty}+C\right)$, and

(b) $N(\bar{I}) \supset N(f)$.

If the above fact is proved, applying it again, we get a Blaschke product $J$ such that

$\left(\mathrm{a}^{\prime}\right) \bar{J}\left|\operatorname{supp} \mu_{x} \in H^{\infty}\right| \operatorname{supp} \mu_{x}$ or $\bar{I}\left|\operatorname{supp} \mu_{x} \in H^{\infty}\right| \operatorname{supp} \mu_{x}$ for every $x \in$ $M\left(H^{\infty}+C\right)$, and

(b') $N(\bar{J}) \supset N(g)$. 
Then by Lemma $2.3, N(\bar{I}) \cap N(\bar{J})=\varnothing$, so we get our assertion.

Using Lemma 2.5, we shall show the existence of a Blaschke product $I$ satisfying (a) and (b). By Lemma 2.2, there is a sequence of interpolating Blaschke products $\left\{I_{n}\right\}_{n=1}^{\infty}$ such that

$$
\left[H^{\infty}, f\right]=\left[H^{\infty},\left\{\bar{I}_{n}\right\}_{n=1}^{\infty}\right]
$$

We note that if $f\left|\operatorname{supp} \mu_{x} \in H^{\infty}\right| \operatorname{supp} \mu_{x}$ for some $x \in M\left(H^{\infty}+C\right)$, then we get $\bar{I}_{n}\left|\operatorname{supp} \mu_{x} \in H^{\infty}\right| \operatorname{supp} \mu_{x}$ for all $n$. Let $\left\{z_{n, k}\right\}_{k=1}^{\infty}$ be the zero sequence of $I_{n}$. Replacing $I_{n}$ by $I_{n}^{\prime}$ such that $I_{n} \bar{I}_{n}^{\prime}$ is a finite Blaschke product, we may assume that

$$
\sum_{n=1}^{\infty} \sum_{k=1}^{\infty}\left(1-\left|z_{n, k}\right|\right)<\infty .
$$

Then $\prod_{n=1}^{\infty} I_{n}$ is a Blaschke product. By our assumption, $\left\{I_{n}\right\}_{n=1}^{\infty}$ and $g$ satisfy the assumptions of Lemma 2.5. Hence there is a Blaschke product $I$ satisfying (a) and $N(\bar{I}) \supset N\left(\bar{I}_{n}\right)$ for all $n$. Since $N(f)$ coincides with the closure of $\bigcup\left\{N\left(\bar{I}_{n}\right) ; n=\right.$ $1,2, \ldots\}$, we get (b). This completes the proof.

To prove the corollaries, we give two lemmas.

LEMMA 2.6 (Sarason's unpublished result, see [8, Theorem 2.8]). Let $f \in L^{\infty}$ with $f^{2}=f$, and let $Q$ be a $Q C$-level set. If $f\left|Q \in H^{\infty}\right| Q$, then $f \mid Q$ is a constant.

LEMMA 2.7. Let $b$ be a sparse Blaschke product with zeros $\left\{w_{n}\right\}_{n=1}^{\infty}$ and $I$ be an inner function. Then $N(\bar{b}) \cap N(\bar{I})=\varnothing$ if and only if $\left|I\left(w_{n}\right)\right| \rightarrow 1(n \rightarrow \infty)$.

Proof. Suppose $N(\bar{b}) \cap N(\bar{I})=\varnothing$. Then $|I|=1$ on $Z(b)$. Since $Z(b)=$ $\operatorname{cl}\left\{w_{n}\right\}_{n=1}^{\infty} \backslash\left\{w_{n}\right\}_{n=1}^{\infty},\left|I\left(w_{n}\right)\right| \rightarrow 1(n \rightarrow \infty)$. Next suppose that $\left|I\left(w_{n}\right)\right| \rightarrow 1(n \rightarrow$ $\infty)$. Then $|I|=1$ on $Z(b)$. Let $x \in M\left(H^{\infty}+C\right)$ with $|b(x)|<1$. Then there is a point $x_{0}$ in $Z(b)$ with $\operatorname{supp} \mu_{x_{0}}=\operatorname{supp} \mu_{x}$ by the proof of Lemma 1 in [9]. Since $\left|I\left(x_{0}\right)\right|=1$, we have $|I(x)|=1$. Thus

$$
\left\{x \in M\left(H^{\infty}+C\right) ;|I(x)|<1\right\} \cap\left\{y \in M\left(H^{\infty}+C\right) ;|b(y)|<1\right\}=\varnothing .
$$

By Lemma 2.3, we have $N(\bar{b}) \cap N(\bar{I})=\varnothing$.

The following corollary shows that $N(f)$ consists of $Q C$-level sets, which is a generalization of Theorem 1 in $[\mathbf{1 3}]$.

Corollary 2.1. For $f \in L^{\infty}, N(f)=\pi^{-1}(\pi(N(f)))$ and $N(f)$ is a weak peak set for $Q A$.

Proof. The inclusion $N(f) \subset \pi^{-1}(\pi(N(f)))$ is trivial. Suppose that $N(f) \varsubsetneqq$ $\pi^{-1}(\pi(N(f)))$. Then there is a $Q C$-level set $Q$ with $N(f) \cap Q \neq \varnothing$ and $Q \not \subset$ $N(f)$. Take an open and closed subset $U$ of $X$ with $U \cap N(f)=\varnothing$ and $U \cap Q \neq$ $\varnothing$. Then $f$ and $\chi_{U}$, the characteristic function of $U$, satisfy the assumption of Theorem 2.1. Thus $N(f) \cap N\left(\chi_{U}\right)=\varnothing$. By Lemma 2.6, $\chi_{U}\left|Q \notin H^{\infty}\right| Q$. Since $Q$ is a weak peak set for $H^{\infty}$, there is $x \in M\left(H^{\infty}+C\right)$ such that supp $\mu_{x} \subset Q$ and $\chi_{U}\left|\operatorname{supp} \mu_{x} \notin H^{\infty}\right| \operatorname{supp} \mu_{x}$. Thus $N\left(\chi_{U}\right) \cap Q \neq \varnothing$. But this contradicts $N(f) \cap Q \neq \varnothing$ and $N(f) \cap N\left(\chi_{U}\right)=\varnothing$. Thus $N(f)=\pi^{-1}(\pi(N(f)))$. By Wolff's theorem [23, Theorem 1 and Lemma 2.3] as the proof of Theorem 1 in $[\mathbf{1 3}], N(f)$ is a weak peak set for $Q A$.

The following follows Corollary 2.1. 
COROLlaRY 2.2. For $f \in L^{\infty}, Q(f) \subset N(f)$ and $\operatorname{cl} Q(f)=N(f)$.

For $f \in L^{\infty}$, we put $Q_{0}(f)=\bigcup\left\{\pi^{-1}(y) ; y \in M(Q C)\right.$ and $f \mid \pi^{-1}(y)$ is not constant\}.

Corollary 2.3. For $f \in L^{\infty}, Q(f) \cup Q(\bar{f}) \subset Q_{0}(f) \subset N(f) \cup N(\bar{f})$.

Proof. By the definitions, $Q(f) \cup Q(\bar{f}) \subset Q_{0}(f)$. Suppose that $Q_{0}(f) \not \subset$ $N(f) \cup N(\bar{f})$. By Corollary 2.1, there is a $Q C$-level set $Q$ with $Q \cap(N(f) \cup N(\bar{f}))=\varnothing$ and $Q \subset Q_{0}(f)$. Take a function $q$ in $Q C$ such that $q=1$ on $Q$ and $q=0$ on $N(f) \cup N(\bar{f})$. By $[\mathbf{2 0}], f q \in Q C$, so $f q$ is constant on $Q$. Thus $f$ is constant on $Q$. This fact contradicts $Q \subset Q_{0}(f)$.

REMARK. In $\S 6$, we will prove that $Q(f)=N(f)$ if and only if $\left[H^{\infty}, f\right]$ is singly generated. If $f \in H^{\infty}, Q(\bar{f}) \subset Q_{0}(\bar{f}) \subset N(\bar{f})$ by Corollary 2.3. Moreover if there is a $Q C$-level set $Q$ such that $f \mid Q$ is real nonconstant, then $Q(\bar{f}) \varsubsetneqq Q_{0}(\bar{f})$, and $\left[H^{\infty}, \bar{f}\right]$ is not singly generated.

COROLlary 2.4. Let $f \in L^{\infty}$. If $I$ is an interpolating Blaschke product with $N(\bar{I}) \subset N(f)$, then $\bar{I} \in\left[H^{\infty}, f\right]$.

Proof. Suppose $\bar{I} \notin\left[H^{\infty}, f\right]$. Then there is a point $x_{0}$ in $M\left(\left[H^{\infty}, f\right]\right)$ with $I\left(x_{0}\right)=0$. Let $\left\{w_{k}\right\}_{k=1}^{\infty}$ be the zero sequence of $I$. Then $x_{0} \in \operatorname{cl}\left\{w_{k}\right\}_{k=1}^{\infty}$. By Lemma 2.2, $\left[H^{\infty}, f\right]=\left[H^{\infty},\left\{\bar{I}_{n}\right\}_{n=1}^{\infty}\right]$ for some sequence of interpolating Blaschke products $\left\{I_{n}\right\}_{n=1}^{\infty}$. Since $\left|I_{n}\left(x_{0}\right)\right|=1$, there is a subsequence $\left\{w_{j_{k}}\right\}_{k=1}^{\infty}$ of $\left\{w_{k}\right\}_{k=1}^{\infty}$ such that $\left|I_{n}\left(w_{j_{k}}\right)\right| \rightarrow 1(k \rightarrow \infty)$ for every $n$. Taking again its subsequence, we may assume that $\left\{w_{j_{k}}\right\}_{k=1}^{\infty}$ is a sparse sequence. Let $b$ be the sparse Blaschke product with zeros $\left\{w_{j_{k}}\right\}_{k=1}^{\infty}$. By Lemma 2.7, $N(\bar{b}) \cap N\left(\bar{I}_{n}\right)=\varnothing$ for every $n$. Hence $\bar{b}$ and $f$ satisfy the assumption of Theorem 2.1. Then $N(\bar{b}) \cap N(f)=\varnothing$. This contradicts $N(\bar{I}) \subset N(f)$, because $N(\bar{b}) \subset N(\bar{I})$.

Corollary 2.5 (CF. [13, COROllary 5]). Let $f$ and $g$ be functions in $L^{\infty}$. Then $N(f) \subset N(g)$ if and only if $\left[H^{\infty}, f\right] \subset\left[H^{\infty}, g\right]$.

Proof. Suppose $N(f) \subset N(g)$. Let $I$ be an interpolating Blaschke product with $\bar{I} \in\left[H^{\infty}, f\right]$. Then $N(\bar{I}) \subset N(f) \subset N(g)$. By Corollary 2.4, we have $\bar{I} \in\left[H^{\infty}, g\right]$. By Chang-Marshall's theorem, $\left[H^{\infty}, f\right] \subset\left[H^{\infty}, g\right]$. The converse assertion is trivial.

For a Douglas algebra $B$, let $N(B)$ equal the closure of

$$
\bigcup\left\{\operatorname{supp} \mu_{x} ; x \in M\left(H^{\infty}+C\right) \backslash M(B)\right\} .
$$

We note that $N\left(\left[H^{\infty}, f\right]\right)=N(f)$.

Corollary 2.6 ( CF. [13, Corollaries 4 AND 6]). Let $B$ be a Douglas algebra.

(i) If $f \in L^{\infty}$ satisfies $N(B) \subset N(f)$, then $B \subset\left[H^{\infty}, f\right]$.

(ii) Let $f \in B$. Then $N(f)=N(B)$ if and only if $B=\left[H^{\infty}, f\right]$. Consequently $B$ is countably generated if and only if there is $f$ in $B$ with $N(f)=N(B)$.

ProOF. (i) Let $I$ be an inner function with $\bar{I} \in B$. Then $N(\bar{I}) \subset N(B) \subset N(f)$. By Corollary 2.4, $\bar{I} \in\left[H^{\infty}, f\right]$. Thus $B \subset\left[H^{\infty}, f\right]$.

(ii) By (i),

$$
N(f)=N(B) \Leftrightarrow B \subset\left[H^{\infty}, f\right] \subset B \Leftrightarrow B=\left[H^{\infty}, f\right] .
$$


3. Geometrical properties of quotient spaces of Douglas algebras. In [14], the author showed that a Douglas algebra $B$ is singly generated if and only if ball $\left(B / H^{\infty}+C\right)$ has extreme points. In this section, we shall prove two theorems as applications of $\S 2$. The first one, Theorem 3.1, is a geometrical characterization of countably generated Douglas algebras. In Theorem 3.2, we shall show that there are no exposed points in ball $\left(B / H^{\infty}+C\right)$. This is already proved in $[\mathbf{1 5}$, Theorem 4] for $B=\left[H^{\infty}, \bar{b}\right]$, where $b$ is a sparse Blaschke product. To state Theorem 3.1, we define an extreme family.

Let $Y$ be a Banach space. If a subset $E$ of ball $Y$ satisfies the following conditions, we shall call it an extreme family;

(a) $\|y\|=1$ for every $y \in E$, and

(b) if a point $y_{0}$ in $Y$ satisfies $\left\|y \pm y_{0}\right\| \leq 1$ for every $y \in E$, then $y_{0}=0$.

By our definition, an extreme family consisting of only one element is an extreme point of ball $Y$.

THEOREM 3.1. Let $B$ be a Douglas algebra with $B \supsetneqq H^{\infty}+C$. Then $B$ is countably generated if and only if $B / H^{\infty}+C$ has an extreme family consisting of countably many elements.

LEMMA $3.1[\mathbf{1 3}$, THEOREM 1]. For an inner function $I$, we have $N(\bar{I})=$ $Q(\bar{I})=\bigcup\left\{Q_{x} ; x \in Z(I)\right\}$.

Proof of TheOrem 3.1. First, suppose that $B=\left[H^{\infty},\left\{\bar{I}_{n}\right\}_{n=1}^{\infty}\right]$ for a sequence of interpolating Blaschke products $\left\{I_{n}\right\}_{n=1}^{\infty}$. It is easy to see

$$
\left\|\bar{I}_{n}+H^{\infty}+C\right\|=1 .
$$

We shall show that $\left\{\bar{I}_{n}+H^{\infty}+C\right\}_{n=1}^{\infty}$ is an extreme family of ball $\left(B / H^{\infty}+C\right)$. Let $g \in B$ with

$$
\left\|\bar{I}_{n} \pm g+H^{\infty}+C\right\| \leq 1 \text { for every } n .
$$

By Corollary 2.1 (or see [13, Theorem 1]), $N\left(\bar{I}_{n}\right)$ is a weak peak set for $Q A$. Then $B_{n}=\left\{f \in L^{\infty} ; f\left|N\left(\bar{I}_{n}\right) \in H^{\infty}\right| N\left(\bar{I}_{n}\right)\right\}$ is a Douglas algebra. By (1), we have $\left\|\bar{I}_{n} \pm g+B_{n}\right\| \leq 1$. By $\left[\mathbf{1 3}\right.$, Theorem 3], $\bar{I}_{n}+B_{n}$ is an extreme point of ball $\left(L^{\infty} / B_{n}\right)$. Thus $g \in B_{n}$, that is,

$$
g\left|N\left(\bar{I}_{n}\right) \in H^{\infty}\right| N\left(\bar{I}_{n}\right) \text { for each } n .
$$

To show $g \in H^{\infty}+C$, let $x \in M\left(H^{\infty}+C\right)$. If $\left|I_{n}(x)\right|=1$ for every $n$, then $x \in M(B)$ and $g\left|\operatorname{supp} \mu_{x} \in H^{\infty}\right| \operatorname{supp} \mu_{x}$. If $\left|I_{n}(x)\right|<1$ for some $n$, then $\operatorname{supp} \mu_{x} \subset N\left(\bar{I}_{n}\right)$. By (2), $g\left|\operatorname{supp} \mu_{x} \in H^{\infty}\right| \operatorname{supp} \mu_{x}$. By $[\mathbf{2 0}]$, we get $g \in H^{\infty}+C$. Thus $\left\{\bar{I}_{n}+H^{\infty}+C\right\}_{n=1}^{\infty}$ is an extreme family.

Next suppose that $B$ is not countably generated. Let $\left\{f_{n}\right\}_{n=1}^{\infty}$ be a sequence in $B$ with $\left\|f_{n}+H^{\infty}+C\right\|=1$. Since $H^{\infty}+C$ has the best approximation property [2], we may assume $\left\|f_{n}\right\|=1$. By Lemma 2.2 , there is a function $F$ in $L^{\infty}$ such that

$$
\left[H^{\infty},\left\{f_{n}\right\}_{n=1}^{\infty}\right]=\left[H^{\infty}, F\right] \subset B .
$$

Since $\left[H^{\infty}, F\right]$ is countably generated by Lemma 2.2 , there is an interpolating Blaschke product $I$ with $\bar{I} \in B$ and $\bar{I} \notin\left[H^{\infty}, F\right]$. By Corollary 2.4, we have 
$N(\bar{I}) \not \subset N(F)$. By Corollary 2.1, there is a $Q C$-level set $Q$ such that $Q \cap N(F)=\varnothing$ and $Q \subset N(\bar{I})$. Then there is a function $q$ in $Q C$ such that

$$
\begin{aligned}
& 0 \leq q \leq 1 \text { and } q=1 \quad \text { on } Q, \\
& q=0 \text { on } N(F) .
\end{aligned}
$$

By Lemma 3.1, we get $\bar{I} q \in B$ and $\bar{I} q \notin H^{\infty}+C$. By (3) and (5), $q f_{n} \in H^{\infty}+C$. Then

$$
\begin{aligned}
\left\|f_{n} \pm \bar{I} q+H^{\infty}+C\right\| & \leq\left\|f_{n} \pm \bar{I} q-q f_{n}\right\| \\
& \leq\||1-q|+|q|\|=1 \quad \text { by }\left\|f_{n}\right\|=1 \text { and (4). }
\end{aligned}
$$

Thus $\left\{f_{n}+H^{\infty}+C\right\}$ is not an extreme family, and this completes the proof.

To prove Theorem 3.2, we need lemmas.

LEMMA 3.2. Let $f \in L^{\infty}$ and $f \notin H^{\infty}+C$. Then $N(f)$ contains uncountably many $Q C$-level sets.

PROOF. By Chang-Marshall's theorem, there is an interpolating Blaschke product $I$ with $\bar{I} \in\left[H^{\infty}, f\right]$. Then $N(\bar{I}) \subset N(f)$. Let $\left\{z_{n}\right\}_{n=1}^{\infty}$ be the zero sequence of I. Take a sparse subsequence $\left\{w_{n}\right\}_{n=1}^{\infty}$ of $\left\{z_{n}\right\}_{n=1}^{\infty}$, and let $b$ be the sparse Blaschke product with zeros $\left\{w_{n}\right\}_{n=1}^{\infty}$. Then $Z(b) \subset Z(I)$. By [13, Lemma 5], $Q_{x} \neq Q_{y}$ for $x, y \in Z(I)$ and $x \neq y$. Since $Z(b)=\operatorname{cl}\left\{w_{n}\right\}_{n=1}^{\infty} \backslash\left\{w_{n}\right\}_{n=1}^{\infty}$ and $\operatorname{cl}\left\{w_{n}\right\}_{n=1}^{\infty}$ is homeomorphic to the Stone-Cech compactification of $\left\{w_{n}\right\}_{n=1}^{\infty}, Z(b)$ is an uncountable set.

The following lemma is a key to prove Theorem 3.2.

LEMMA 3.3. Let I be an interpolating Blaschke product. Let $\mu$ be a probability measure on $N(\bar{I})$. Then $\operatorname{supp} \mu \varsubsetneqq N(\bar{I})$, and there is a sparse Blaschke product $b$ such that $I \bar{b} \in H^{\infty}$ and $N(\bar{b}) \subset N(\bar{I}) \backslash \operatorname{supp} \mu$.

Proof. By Lemma 3.2, $N(\bar{I})$ contains uncountably many $Q C$-level sets. Then there is a $Q C$-level set $Q$ such that $Q \subset N(\bar{I})$ and $\mu(Q)=0$. Since $Q$ is a weak peak set for $Q A$, there is a peak set $E$ for $Q A$ such that

$$
Q \subset E \subset X \text { and } \mu(E)=0 \text {. }
$$

Let $f$ be a peaking function in $Q A$ for $E$, that is,

$$
f=1 \quad \text { on } E \quad \text { and } \quad|f|<1 \text { on } X \backslash E .
$$

We put

$$
K_{n}=\{x \in X ;|f(x)| \leq 1-1 / n\}
$$

Then

$$
\mu\left(K_{n} \cap N(\bar{I})\right)=\mu\left(K_{n}\right) \rightarrow 1 \quad(n \rightarrow \infty) .
$$

By Lemma 3.1, there is a point $x_{0} \in Z(I)$ such that $Q=Q_{x_{0}}$. Take an open and closed subset $U_{n}$ of $Z(I)$ such that

$$
\{x \in Z(I) ;|f(x)| \leq 1-1 / n\} \subset U_{n} \subset\{x \in Z(I) ;|f(x)| \leq 1-1 / n+1\} .
$$

Then $\bigcup\left\{Q_{x} ; x \in U_{n}\right\} \subset K_{n+1}$, because $f \in Q A$ is constant on each $Q C$-level set. Since $U_{n}$ is an open and closed subset of $Z(I)$, there is an interpolating Blaschke product $I_{n}$ with $I \bar{I}_{n} \in H^{\infty}$ and $Z\left(I_{n}\right)=U_{n}[\mathbf{1 2}$, Corollary 1]. By Lemma 3.1,

$$
N\left(\bar{I}_{n}\right) \subset K_{n+1} \text {. }
$$


Moreover we have

$$
K_{n} \cap N(\bar{I}) \subset N\left(\bar{I}_{n}\right) .
$$

To show (6), let $y \in K_{n} \cap N(\bar{I})$. By Lemma 3.1, there is a point $x_{1} \in Z(I)$ such that $y \in Q_{x_{1}}$. Since $|f(y)| \leq 1-1 / n,\left|f\left(x_{1}\right)\right| \leq 1-1 / n$. Thus $x_{1} \in U_{n}$ and $y \in N\left(\bar{I}_{n}\right)$. Since $Q_{x_{0}} \cap N\left(\bar{I}_{n}\right)=\varnothing$ by (1), (2), (3) and (5), we have $\left|I_{n}\left(x_{0}\right)\right|=1$. Since $I\left(x_{0}\right)=0, \bar{I} \notin\left[H^{\infty},\left\{\bar{I}_{n}\right\}_{n=1}^{\infty}\right]$. By the proof of Corollary 2.4, there is a sparse Blaschke product $b$ such that

$$
I \bar{b} \in H^{\infty} \quad \text { and } \quad N(\bar{b}) \cap \operatorname{cl}\left(\bigcup\left\{N\left(\bar{I}_{n}\right) ; n=1,2, \ldots\right\}\right)=\varnothing .
$$

Since $I \bar{b} \in H^{\infty}, N(\bar{b}) \subset N(\bar{I})$. By equations (4) and (6), we have supp $\mu \subset$ $\operatorname{cl}\left(\bigcup\left\{N\left(\bar{I}_{n}\right) ; n=1,2, \ldots\right\}\right)$. Thus we get our assertions.

THEOREM 3.2. Let $B$ be a Douglas algebra with $B \supsetneqq H^{\infty}+C$. Then there are no exposed points in ball $\left(B / H^{\infty}+C\right)$.

ProOF. By $[\mathbf{1 4}]$ and Lemma 2.1, we may assume $B=\left[H^{\infty}, \bar{I}\right]$ for some interpolating Blaschke product $I$. Let $f \in B$ with $\left\|f+H^{\infty}+C\right\|=1$. Since $H^{\infty}+C$ has the best approximation property [2], we may assume $\|f\|=1$. Let $\mu$ be a measure on $X$ such that $\|\mu\|=1, \mu \perp H^{\infty}+C$, and $\int_{X} f d \mu=1$. By [13, Lemma 9], $\operatorname{supp} \mu \subset N(\bar{I})$. By Lemmas 3.1 and 3.3, there is a $Q C$-level set $Q$ with $Q \subset N(\bar{I})$ and $Q \cap \operatorname{supp} \mu=\varnothing$. This fact is the key point to prove a special case of Theorem $3.2[\mathbf{1 3}$, Theorem 3]. We can go the same way as in $[\mathbf{1 3}]$, and we can show the existence of $g$ in $B$ such that $\left\|g+H^{\infty}+C\right\|=1, \int_{X} g d \mu=1$ and $f+H^{\infty}+C \neq$ $g+H^{\infty}+C$. This completes the proof.

4. Sarason's three functions problem. In [22], Sarason showed that if $f$ and $g$ in $L^{\infty}$ satisfy $f\left|\operatorname{supp} \mu_{x} \in H^{\infty}\right| \operatorname{supp} \mu_{x}$ or $g\left|\operatorname{supp} \mu_{x} \in H^{\infty}\right| \operatorname{supp} \mu_{x}$ for every $x \in M\left(H^{\infty}+C\right)$, then $f\left|Q \in H^{\infty}\right| Q$ or $g\left|Q \in H^{\infty}\right| Q$ for every $Q C$-level set $Q$. The following problem occurs from the above fact [22]; is it still true for three functions in $L^{\infty}$ ? In this section, we shall show

THEOREM 4.1. Let $\left\{f_{n}\right\}_{n=1}^{N}$ be a finite subset of $L^{\infty}$. Suppose that for each point $x \in M\left(H^{\infty}+C\right)$, there exists $n$ such that $f_{n}\left|\operatorname{supp} \mu_{x} \in H^{\infty}\right| \operatorname{supp} \mu_{x}$. Then $\bigcap_{n=1}^{N} N\left(f_{n}\right)=\varnothing$.

We note that Corollary 2.1 and Theorem 4.1 give an affirmative answer for the above problem. To show Theorem 4.1, we need some lemmas.

LEMMA 4.1. Let $B$ be a Douglas algebra. Then $B$ is countably generated if and only if $M(B)$ is a $G_{\delta}$-subset of $M\left(H^{\infty}\right)$. Then

PROOF. Let $B=\left[H^{\infty},\left\{\bar{I}_{n}\right\}_{n=1}^{\infty}\right]$ for a sequence of inner functions $\left\{I_{n}\right\}_{n=1}^{\infty}$.

$$
\begin{aligned}
M(B) & =\left\{x \in M\left(H^{\infty}\right) ;\left|I_{n}(x)\right|=1 \text { for every } n\right\} \\
& =\bigcap_{n=1}^{\infty}\left\{x \in M\left(H^{\infty}\right) ;\left|I_{n}(x)\right|=1\right\} .
\end{aligned}
$$

It is easy to see that $M(B)$ is a $G_{\delta}$-subset of $M\left(H^{\infty}\right)$. 
Suppose that $M(B)$ is a $G_{\delta}$-subset of $M\left(H^{\infty}\right)$. Then there is a sequence of open subsets $\left\{U_{n}\right\}_{n=1}^{\infty}$ of $M\left(H^{\infty}\right)$ with $\bigcap_{n=1}^{\infty} U_{n}=M(B)=\bigcap_{\bar{I} \in B}\left\{x \in M\left(H^{\infty}\right) ;|I(x)|\right.$ $=1\}$, where $I$ runs through all inner functions with $\bar{I} \in B$. Since $U_{n}^{c} \subset$ $M\left(H^{\infty}\right) \backslash M(B)$ and $U_{n}^{c}$ is a compact subset of $M\left(H^{\infty}\right)$, there is an inner function $I_{n}$ such that $\bar{I}_{n} \in B$ and $U_{n}^{c} \subset\left\{x \in M(B) ;\left|I_{n}(x)\right|<1\right\}$. Then $M(B)=$ $M\left(\left[H^{\infty},\left\{\bar{I}_{n}\right\}_{n=1}^{\infty}\right]\right.$. By Chang-Marshall's theorem, we obtain $B=\left[H^{\infty},\left\{\bar{I}_{n}\right\}_{n=1}^{\infty}\right]$.

LEMMA 4.2 (Sarason's unpublished result, see [7, Theorem 3.4]). Let $\left\{B_{\alpha}\right\}_{\alpha \in \Lambda}$ be a family of Douglas algebras. Then $M\left(\bigcap_{\alpha \in \Lambda} B_{\alpha}\right)$ coincides with the closure of $\bigcup_{\alpha \in \Lambda} M\left(B_{\alpha}\right)$ in $M\left(H^{\infty}\right)$.

LEMMA 4.3. For functions $f$ and $g$ in $L^{\infty}$, there is a function $h$ in $L^{\infty}$ with $\left[H^{\infty}, h\right]=\left[H^{\infty}, f\right] \cap\left[H^{\infty}, g\right]$.

ProOF. By Lemma 4.2,

$$
M\left(\left[H^{\infty}, f\right] \cap\left[H^{\infty}, g\right]\right)=M\left(\left[H^{\infty}, f\right]\right) \cup M\left(\left[H^{\infty}, g\right]\right) .
$$

By Lemma 2.2 and 4.1, $M\left(\left[H^{\infty}, f\right]\right) \cup M\left(\left[H^{\infty}, g\right]\right)$ is a $G_{\delta}$-subset of $M\left(H^{\infty}\right)$, so is $M\left(\left[H^{\infty}, f\right] \cap\left[H^{\infty}, g\right]\right)$. By Lemmas 2.2 and 4.1 again, there is $h \in L^{\infty}$ with $\left[H^{\infty}, h\right]=\left[H^{\infty}, f\right] \cap\left[H^{\infty}, g\right]$.

LEMMA 4.4. Let $f, g$ and $h$ be functions in $L^{\infty}$ with $\left[H^{\infty}, h\right]=\left[H^{\infty}, f\right] \cap$ $\left[H^{\infty}, g\right]$. Then $N(h)=N(f) \cap N(g)$.

ProOF. By our assumption, we have easily $N(h) \subset N(f) \cap N(g)$. Suppose that $N(h) \varsubsetneqq N(f) \cap N(g)$. By Corollary 2.1, there is a $Q C$-level set $Q$ with $Q \subset N(f) \cap N(g)$ and $Q \cap N(h)=\varnothing$. Take a function $q$ in $Q C$ such that

(1) $0 \leq q \leq 1$ on $X$ and $q=0$ on $N(h)$, and

(2) $q=1$ on some open neighborhood of $Q$.

By Lemma 4.2,

$$
M\left(\left[H^{\infty}, h\right]\right)=M\left(\left[H^{\infty}, f\right]\right) \cup M\left(\left[H^{\infty}, g\right]\right) .
$$

By (1) and (3), we have $f q\left|\operatorname{supp} \mu_{x} \in H^{\infty}\right| \operatorname{supp} \mu_{x}$ or $g q\left|\operatorname{supp} \mu_{x} \in H^{\infty}\right| \operatorname{supp} \mu_{x}$ for every $x \in M\left(H^{\infty}+C\right)$. By Theorem 2.1, we get $N(f q) \cap N(g q)=\varnothing$. By (2), $Q \cap N(f(1-q))=\varnothing$. Since $N(f)=N(f q) \cup N(f(1-q)), Q \subset N(f q)$. Also we obtain $Q \subset N(g q)$. These contradict $N(f q) \cap N(g q)=\varnothing$.

Proof OF TheOREM 4.1. By Lemmas 4.3 and 4.4 , there is $F \in L^{\infty}$ such that $\left[H^{\infty}, F\right]=\bigcap_{n=1}^{N}\left[H^{\infty}, f_{n}\right]$ and $N(F)=\bigcap_{n=1}^{N} N\left(f_{n}\right)$. By Lemma 4.2 and our assumption, $F \in H^{\infty}+C$. Thus $N(F)=\varnothing$. This completes the proof.

We note that there is a sequence of functions $\left\{f_{n}\right\}_{n=1}^{\infty}$ in $L^{\infty}$ such that and

(a) for each $x$ in $M\left(H^{\infty}+C\right)$, there exists $n$ such that $f_{n}\left|\operatorname{supp} \mu_{x} \in H^{\infty}\right| \operatorname{supp} \mu_{x}$,

(b) $\bigcap_{n=1}^{\infty} N\left(f_{n}\right) \neq \varnothing$.

EXAMPLE. Let $\lambda_{n} \in \partial D$ with $\lambda_{n} \rightarrow 1(n \rightarrow \infty)$, and let $S_{n}$ be the singular inner function associated with the singular measure $\sum_{k=n}^{\infty}(1 / 2)^{k} \delta_{\lambda_{k}}$. We put $f_{n}=$ $(z-1) S_{n}$, then $\left\{f_{n}\right\}_{n=1}^{\infty}$ satisfies (a). Since $N\left(f_{n}\right) \supset N\left(f_{n+1}\right)$, we get $\bigcap_{n=1}^{\infty} N\left(f_{n}\right)$ $\neq \varnothing$. We note that if $Q \subset \bigcap_{n=1}^{\infty} N\left(f_{n}\right)$ then $f_{n}\left|Q \in H^{\infty}\right| Q$ for every $n$, hence for each $Q C$-level set $Q$ there is $n$ such that $f_{n}\left|Q \in H^{\infty}\right| Q$.

In the last part of this section, we give a result which relates to Corollary 2.6. 
Proposition 4.1. For every $f \in L^{\infty}$ with $N(f) \neq \varnothing$, there is a Douglas algebra $B$ such that

(i) $N(B)=N(f)$, and

(ii) $B$ is not countably generated.

Proof. Let $Q$ be a $Q C$-level set with $f\left|Q \notin H^{\infty}\right| Q$. Put

$B=\left[H^{\infty}, \bar{I} ; I\right.$ is an inner function with $\bar{I} \in\left[H^{\infty}, f\right]$ and $\left.\bar{I}\left|Q \in H^{\infty}\right| Q\right]$.

Then $B \subset\left[H^{\infty}, f\right]$, so $N(B) \subset N(f)$.

Claim. Put $E=\bigcup\left\{\operatorname{supp} \mu_{x} ; x \in M\left(H^{\infty}+C\right), f\left|\operatorname{supp} \mu_{x} \notin H^{\infty}\right| \operatorname{supp} \mu_{x}\right.$ and supp $\left.\mu_{x} \cap Q=\varnothing\right\}$. Then $E$ is dense in $N(f)$.

To show our claim, suppose not. Then $\operatorname{cl} E \not \supset$ supp $\mu_{y}$ for some $y \in M\left(H^{\infty}+C\right)$ with $f\left|\operatorname{supp} \mu_{y} \notin H^{\infty}\right| \operatorname{supp} \mu_{y}$ and supp $\mu_{y} \subset Q$. Hence there is an open and closed subset $U$ of $X$ such that $E \cap U=\varnothing$, supp $\mu_{y} \cap U \neq \varnothing$ and supp $\mu_{y} \not \subset U$. By Lemma 2.6, $Q_{y} \subset N\left(\chi_{U}\right)$. Thus $N(f) \cap N\left(\chi_{U}\right) \neq \varnothing$. By Lemma 4.3, there is $h \in L^{\infty}$ such that $\left[H^{\infty}, h\right]=\left[H^{\infty}, f\right] \cap\left[H^{\infty}, \chi_{U}\right]$. By Lemma 4.4, $h \notin H^{\infty}+C$. By Lemma 3.2, there is $\zeta \in M\left(H^{\infty}+C\right)$ with $h\left|\operatorname{supp} \mu_{\zeta} \notin H^{\infty}\right| \operatorname{supp} \mu_{\zeta}$ and $\operatorname{supp} \mu_{\zeta} \cap Q=\varnothing$. By Lemma 4.2 , both $f \mid \operatorname{supp} \mu_{\zeta}$ and $\chi_{U} \mid \operatorname{supp} \mu_{\zeta}$ are not contained in $H^{\infty} \mid \operatorname{supp} \mu_{\zeta}$. This contradicts the definitions of $E$ and $U$. Hence we get our claim.

To show (i), it is sufficient to prove $E \subset N(B)$ by our claim. To prove this, let $x \in M\left(H^{\infty}+C\right)$ such that $f\left|\operatorname{supp} \mu_{x} \notin H^{\infty}\right| \operatorname{supp} \mu_{x}$ and supp $\mu_{x} \cap Q=\varnothing$. Take a function $q$ in $Q C$ with $q=0$ on $Q$ and $q=1$ on $\operatorname{supp} \mu_{x}$. Then $\left[H^{\infty}, f q\right] \subset B$. Since $f q\left|\operatorname{supp} \mu_{x} \notin H^{\infty}\right| \operatorname{supp} \mu_{x}, x \notin M(B)$. Hence supp $\mu_{x} \subset N(B)$, so $E \subset N(B)$.

To show (ii), suppose not. Then $B=\left[H^{\infty}, F\right]$ for some $F \in\left[H^{\infty}, f\right]$. By Corollary 2.6, $\left[H^{\infty}, F\right]=\left[H^{\infty}, f\right]$. Since $F\left|Q \in H^{\infty}\right| Q, f\left|Q \in H^{\infty}\right| Q$. But this is a contradiction.

5. Discrete sequences in $M(Q C)$. A sequence $\left\{y_{n}\right\}_{n=1}^{\infty}$ in a topological space $Y$ is called discrete if there is a sequence of open subsets $\left\{V_{n}\right\}_{n=1}^{\infty}$ of $Y$ such that $Y_{n} \in V_{n}$ and $V_{n} \cap \mathrm{cl}\left(\bigcup_{m \neq n} V_{n}\right)=\varnothing$. In this section, we study discrete sequences in $M(Q C)$ and show three theorems as applications of $\S 2$. The first one, Theorem 5.1 , gives properties of a sequence of $Q C$-level sets. In Theorem 5.2, we shall show the existence of a certain function in $H^{\infty}$, which is motivated by [11]. Using them, we shall prove a theorem which is more precise than the one proved in $[8$, Theorem 2.1].

A $Q C$-level set is called simple if it consists of only one point. It is not known whether there is a simple $Q C$-level set or not. It is easy to see that a $Q C$-level set $Q$ is not simple if and only if there is $x \in M\left(H^{\infty}+C\right) \backslash X$ such that supp $\mu_{x} \subset Q$. We note that every $Q C$-level set in $N(f), f \in L^{\infty}$, is not simple. Because, for a given $f \in L^{\infty}$, there is an inner function $I$ such that $N(f) \subset N(\bar{I})$ (see the proof of Corollary 7 in [13]). By Lemma 3.1, $N(\bar{I})$ does not contain any simple $Q C$-level sets.

A discrete sequence $\left\{y_{n}\right\}_{n=1}^{\infty}$ in $M(Q C)$ is called strongly discrete if each $\pi^{-1}\left(y_{n}\right)$ is not simple.

THEOREM 5.1. Let $\left\{y_{n}\right\}_{n=1}^{\infty}$ be a strongly discrete sequence in $M(Q C)$, and let $y_{0} \in M(Q C)$ be its cluster point. Then

(i) $\pi^{-1}\left(y_{0}\right)$ is not simple.

(ii) $\pi^{-1}\left(y_{0}\right) \subset \operatorname{cl}\left(\bigcup_{n=1}^{\infty} \pi^{-1}\left(y_{n}\right)\right)$. 
(iii) If $\left\{a_{n}\right\}_{n=1}^{\infty}$ is a bounded sequence of complex numbers, there is $h$ in $Q A$ such that $h\left(y_{n}\right)=a_{n}$ for every $n$.

Proof. By our assumption, there is a sequence of open subsets $\left\{V_{n}\right\}_{n=1}^{\infty}$ of $M(Q C)$ satisfying $y_{n} \in V_{n}$ and

$$
V_{n} \cap \mathrm{cl}\left(\bigcup_{m \neq n} V_{m}\right)=\varnothing .
$$

Let $\left\{x_{n}\right\}_{n=1}^{\infty}$ be a sequence in $M\left(H^{\infty}+C\right) \backslash X$ with supp $\mu_{x_{n}} \subset \pi^{-1}\left(y_{n}\right)$. By [10, p. 177], there is an inner function $I_{n}$ with

$$
\left|I_{n}\left(x_{n}\right)\right|<1 .
$$

Take a function $q_{n}$ in $Q C$ such that $\left\|q_{n}\right\|=1$,

$$
q_{n}\left(y_{n}\right)=1 \quad \text { and } \quad q_{n}=0 \quad \text { on } M(Q C) \backslash V_{n} .
$$

Put $F=\sum_{n=1}^{\infty}(1 / 2)^{n} \bar{I}_{n} q_{n}$. Then $F \in L^{\infty}$. By (1), (2) and (3), $F \mid \operatorname{supp} \mu_{x_{n}} \notin$ $H^{\infty} \mid \operatorname{supp} \mu_{x_{n}}$. Hence $\operatorname{supp} \mu_{x_{n}} \subset N(F)$, so $y_{n} \in \pi(N(F))$ for every $n$. Thus $\operatorname{cl}\left\{y_{n}\right\}_{n=1}^{\infty} \subset \pi(N(F))$. Since $\pi(N(F))$ is a compact subset of $M(Q C), y_{0} \in$ $\pi(N(F))$. By Corollary 2.1, $\pi^{-1}\left(y_{0}\right) \subset N(F)$. By the remark before Theorem 5.1 , we get (i).

To show (ii), suppose that $\pi^{-1}\left(y_{0}\right) \not \subset \mathrm{cl}\left(\bigcup_{n=1}^{\infty} \pi^{-1}\left(y_{n}\right)\right)$. There is an open and closed subset $U$ of $X$ such that $U \cap \operatorname{cl}\left(\bigcup_{n=1}^{\infty} \pi^{-1}\left(y_{n}\right)\right)=\varnothing$ and $U \cap \pi^{-1}\left(y_{0}\right) \neq \varnothing$. Hence we may take a sequence of open subsets $\left\{V_{n}\right\}_{n=1}^{\infty}$ satisfying moreover

$$
\pi^{-1}(U) \cap V_{n}=\varnothing \text {. }
$$

By the same way as (i), we have a function $F=\sum_{n=1}^{\infty}(1 / 2)^{n} \bar{I}_{n} q_{n}$. Let $x \in$ $M\left(H^{\infty}+C\right)$ with $F\left|\operatorname{supp} \mu_{x} \notin H^{\infty}\right| \operatorname{supp} \mu_{x}$. Then $\bar{I}_{n} q_{n}\left|\operatorname{supp} \mu_{x} \notin H^{\infty}\right| \operatorname{supp} \mu_{x}$ for some $n$. By (3), supp $\mu_{x} \subset \pi^{-1}\left(V_{n}\right)$. By (4), supp $\mu_{x} \subset X \backslash U$. Hence we have $N(F) \subset X \backslash U$. Since $\pi^{-1}\left(y_{0}\right) \subset N(F)$ by the proof of (i), we get $\pi^{-1}\left(y_{0}\right) \cap U=\varnothing$. But this is a contradiction, so we get (ii).

(iii) Let $F$ be a function in the proof of (i). By [23, Lemmas 2.2 and 2.3], $m_{0}(\pi(N(F)))=0$ and $\pi(N(F))$ is an interpolation set for $Q A$, that is, $Q A \mid \pi(N(F))$ $=C(\pi(N(F)))$. Since $\operatorname{cl}\left\{y_{n}\right\}_{n=1}^{\infty} \subset \pi(N(F)), \operatorname{cl}\left\{y_{n}\right\}_{n=1}^{\infty}$ is an interpolation set for $Q A$. To prove (iii), it is sufficient to show that $\operatorname{cl}\left\{y_{n_{k}}\right\}_{k=1}^{\infty} \cap \operatorname{cl}\left(\left\{y_{n}\right\}_{n=1}^{\infty} \backslash\left\{y_{n_{k}}\right\}_{k=1}^{\infty}\right)$ $=\varnothing$ for every subset $\left\{y_{n_{k}}\right\}_{k=1}^{\infty}$ of $\left\{y_{n}\right\}_{n=1}^{\infty}$ (see $[10$, p. 205]). To show this, put $G=\sum_{k=1}^{\infty}(1 / 2)^{k} \bar{I}_{n_{k}} q_{n_{k}}$ and $H=F-G$. By our construction, $G$ and $H$ satisfy the assumption of Theorem 2.1, so we get $N(G) \cap N(H)=\varnothing$. Since $\left\{y_{n_{k}}\right\}_{k=1}^{\infty} \subset$ $\pi(N(G))$ and $\left\{y_{n}\right\}_{n=1}^{\infty} \backslash\left\{y_{n_{k}}\right\}_{k=1}^{\infty} \subset \pi(N(H))$, we have

$$
\begin{aligned}
\operatorname{cl}\left\{y_{n_{k}}\right\}_{k=1}^{\infty} \cap \operatorname{cl}\left(\left\{y_{n}\right\}_{n=1}^{\infty} \backslash\left\{y_{n_{k}}\right\}_{k=1}^{\infty}\right) & \subset \pi(N(G)) \cap \pi(N(H)) \\
& =\pi(N(G) \cap N(H)) \text { by Corollary } 2.1 \\
& =\varnothing .
\end{aligned}
$$

This completes the proof.

In [11], Hoffman showed that a discrete sequence $\left\{y_{n}\right\}_{n=1}^{\infty}$ in $X$ is an $l^{\infty}$. interpolation set for $H^{\infty}$, that is, for every bounded sequence of complex numbers $\left\{a_{n}\right\}_{n=1}^{\infty}$ there is $h \in H^{\infty}$ such that $h\left(y_{n}\right)=a_{n}$ for $n=1,2, \ldots$. Using his technique, we shall show the existence of a certain function in $H^{\infty}$. 
LEMMA 5.1 [11]. Let $K$ be a closed subset of $X$ with $m(K)=0$. Let $g$ be a bounded continuous function on $X \backslash K$. Suppose that there is a bounded sequence $\left\{f_{n}\right\}_{n=1}^{\infty}$ in $H^{\infty}$ such that $f_{n}$ converges to $g$ uniformly on each compact subset of $X \backslash K$. Then there is $f \in H^{\infty}$ with $f \mid X \backslash K=g$.

THEOREM 5.2. Let $\left\{y_{n}\right\}_{n=1}^{\infty}$ be a strongly discrete sequence in $M(Q C)$. Let $\left\{h_{n}\right\}_{n=1}^{\infty}$ be a bounded sequence in $H^{\infty}+C$. Then there exists a function $F$ in $H^{\infty}$ such that $F=h_{n}$ on $\pi^{-1}\left(y_{n}\right)$ for every $n$.

Proof. Suppose that $\left\|h_{n}\right\|<M$, where $M$ is an absolute constant. Since $\pi^{-1}\left(y_{n}\right)$ is a weak peak set for $H^{\infty}$ and $H^{\infty}+C\left|\pi^{-1}\left(y_{n}\right)=H^{\infty}\right| \pi^{-1}\left(y_{n}\right)$, there is $f_{n} \in H^{\infty}$ such that $f_{n}\left|\pi^{-1}\left(y_{n}\right)=h_{n}\right| \pi^{-1}\left(y_{n}\right)$ and $\left\|f_{n}\right\|<M$. Let $\left\{V_{n}\right\}_{n=1}^{\infty}$ be a sequence of open subsets of $M(Q C)$ such that $y_{n} \in V_{n}$ and $V_{n} \cap \operatorname{cl}\left(\bigcup_{m \neq n} V_{m}\right)=$ $\varnothing$. Let $W_{0}$ be the interior of $\pi^{-1}\left(M(Q C) \backslash \bigcup_{n=1}^{\infty} V_{n}\right)$. By $\left[4\right.$, p. 18], $m\left(W_{0}\right)=$ $m\left(\pi^{-1}\left(M(Q C) \backslash \bigcup_{n=1}^{\infty} V_{n}\right)\right)$. Put $K=\left(X \backslash W_{0}\right) \backslash \bigcup_{n=1}^{\infty} \pi^{-1}\left(V_{n}\right)$. Then $K$ is a compact subset of $X$ and $m(K)=0$, because

$$
\begin{aligned}
m(K) & =1-m\left(W_{0}\right)-m\left(\bigcup_{n=1}^{\infty} \pi^{-1}\left(V_{n}\right)\right) \\
& =1-m\left(\pi^{-1}\left(M(Q C) \backslash \bigcup_{n=1}^{\infty} V_{n}\right)\right)-m\left(\pi^{-1}\left(\bigcup_{n=1}^{\infty} V_{n}\right)\right) \\
& =1-m_{0}\left(M(Q C) \backslash \bigcup_{n=1}^{\infty} V_{n}\right)-m_{0}\left(\pi^{-1}\left(\bigcup_{n=1}^{\infty} V_{n}\right)\right)=0 .
\end{aligned}
$$

We may take a function $q_{n}$ in $Q A$ satisfying

(1) $\left\|q_{n}\right\|=1, q_{n}\left(y_{n}\right)=1$ and $\left|q_{n}\right|<(1 / 2)^{n}$ on $M(Q C) \backslash V_{n}$.

By (iii) of Theorem 5.1, we may assume that

(2) $q_{m}\left(y_{n}\right)=0$ if $m \neq n$.

Put $G_{N}=\sum_{k=1}^{N} f_{k} q_{k}$. Then $G_{N} \in H^{\infty}$. We shall show that $\left\{G_{N}\right\}_{N=1}^{\infty}$ satisfies the assumption of Lemma 5.1 for $K$. By (1), we have

$$
\begin{aligned}
& \text { on } \pi^{-1}\left(V_{n}\right), \quad\left|G_{N}\right| \leq\left|f_{n}\right|+\sum_{k \neq n}\left|f_{k}\right|\left|q_{k}\right| \\
& \leq M\left(1+\sum_{k=1}^{\infty}\left(\frac{1}{2}\right)^{k}\right) \leq 2 M \\
& \text { on }(X \backslash K) \backslash \bigcup_{n=1}^{\infty} \pi^{-1}\left(V_{n}\right), \quad\left|G_{N}\right| \leq M \sum_{k=1}^{\infty}\left(\frac{1}{2}\right)^{k} \leq M
\end{aligned}
$$

Hence $\left\{G_{N}\right\}_{N=1}^{\infty}$ is a bounded sequence in $H^{\infty}$. Let $E$ be a compact subset of $X \backslash K$. Then $K \subset W_{0} \cup \bigcup_{k=1}^{n_{0}} \pi^{-1}\left(V_{k}\right)$ for some $n_{0}$. For $n_{0} \leq i<j$, we have

$$
\begin{aligned}
\left|G_{j}-G_{i}\right| & =\left|\sum_{k=i+1}^{j} f_{k} q_{k}\right| \\
& \leq M \sum_{k=i+1}^{j}\left(\frac{1}{2}\right)^{k} \leq M\left(\frac{1}{2}\right)^{i} \text { on } W_{0} \cup \bigcup_{k=1}^{n_{0}} \pi^{-1}\left(V_{k}\right) .
\end{aligned}
$$


Hence $\left\{G_{N}\right\}_{N=1}^{\infty}$ converges to $\sum_{k=1}^{\infty} f_{k} q_{k}$ uniformly on $E$. By Lemma 5.1, there is a function $G$ in $H^{\infty}$ such that $F=\sum_{k=1}^{\infty} f_{k} q_{k}$ on $X \backslash K$. By (1) and (2), we get $F\left|\pi^{-1}\left(y_{n}\right)=f_{n}\right| \pi^{-1}\left(y_{n}\right)=h_{n} \mid \pi^{-1}\left(y_{n}\right)$.

A closed subset $E$ of $X$ is called antisymmetric for $H^{\infty}$ if $H^{\infty} \mid E$ does not contain any nonconstant real functions. An antisymmetric set is called maximal if there are no antisymmetric sets which contain $E$ properly.

THEOREM 5.3 (CF. [8, THEOREM 2.1]). Let $\left\{y_{n}\right\}_{n=1}^{\infty}$ be a strongly discrete sequence in $M(Q C)$. Let $\left\{\lambda_{n}\right\}_{n=1}^{\infty}$ be a sequence in $X$ with $\lambda_{n} \in \pi^{-1}\left(y_{n}\right)$. If $\lambda_{0}$ is a cluster point of $\left\{\lambda_{n}\right\}_{n=1}^{\infty}$ in $X$, then $\left\{\lambda_{0}\right\}$ is a maximal antisymmetric set for $H^{\infty}$ and it is not a $Q C$-level set.

PROOF. Let $\lambda_{0}$ be a cluster point of $\left\{\lambda_{n}\right\}_{n=1}^{\infty}$ with $\lambda_{n} \in \pi^{-1}\left(y_{n}\right)$. There is a $Q C$-level set $Q_{0}$ with $Q_{0} \ni \lambda_{0}$. Since $\pi\left(Q_{0}\right) \in \operatorname{cl}\left\{y_{n}\right\}_{n=1}^{\infty}$, there is $y_{0}$ in $\operatorname{cl}\left\{y_{n}\right\}_{n=1}^{\infty}$ such that $Q_{0}=\pi^{-1}\left(y_{0}\right)$. By Theorem 5.1(i), $Q_{0}$ is not simple. We note that the maximal antisymmetric set containing $y_{0}$ is contained in $Q_{0}$. To show our assertion, let $E$ be a closed subset with $\left\{y_{0}\right\} \varsubsetneqq E \subset Q_{0}$. We shall show that $E$ is not antisymmetric. Take an open and closed subset $U$ of $X$ satisfying $\lambda_{0} \in U$ and $E \not \subset U$. By [1], there is $h \in H^{\infty}+C$ such that $|h|=\chi_{U}$ on $X$. Using a function $h$, we shall construct a function $F$ in $H^{\infty}$ such that

(1) $F=1$ on $U \cap\left\{\lambda_{n}\right\}_{n=1}^{\infty}$,

(2) $F=0$ on $U^{c} \cap \pi^{-1}\left\{y_{n}\right\}$ for every $n$, and

(3) the sequence of ranges $F\left(\pi^{-1}\left(y_{n}\right)\right)$ converges in $[-1,1]$, that is, for every open subset $W$ in the complex plane with $[-1,1] \subset W$ there is $n_{0}$ such that $F\left(\pi^{-1}\left(y_{n}\right)\right) \subset$ $W$ for every $n \geq n_{0}$.

We let $D_{n}$ denote the open ellipse with major axis $[-1,1]$ and minor axis $[-i / n, i / n]$. Let $\psi_{n}$ be a conformal mapping of $D$ onto $D_{n}$ such that $\psi_{n}(0)=0$ and $\psi_{n}\left(h\left(\lambda_{n}\right)\right)=1$ for every $n$ with $\left|h\left(\lambda_{n}\right)\right|=1$. We note that $\psi_{n} \circ h \in H^{\infty}+C$ and $\left\|\psi_{n} \circ h\right\|=1$ for every $n$. By Theorem 5.2, there exists a function $F$ in $H^{\infty}$ such that $F\left|\pi^{-1}\left(y_{n}\right)=\psi_{n} \circ h\right| \pi^{-1}\left(y_{n}\right)$. It is easy to see that $F$ satisfies (1) and (2) and (3). Since $\lambda_{0} \in \operatorname{cl}\left\{\lambda_{n}\right\}_{n=1}^{\infty}, F\left(\lambda_{0}\right)=1$ by (1). By Theorem 5.1(ii) and (2), $F=0$ on $U^{c} \cap \pi^{-1}\left\{y_{0}\right\}$. Also by Theorem 5.1(ii) and (3), $F$ is a real function on $\pi^{-1}\left(y_{0}\right)$. Thus $F\left|E \in H^{\infty}\right| E$ is not a nonconstant real function. Hence $E$ is not antisymmetric.

REMARK. It is not true that a cluster point of discrete sequence $\left\{\lambda_{n}\right\}_{n=1}^{\infty}$ in $X$ is a maximal antisymmetric set for $H^{\infty}$. For, let $x \in M\left(H^{\infty}+C\right) \backslash X$, then supp $\mu_{x}$ is an antisymmetric set for $H^{\infty}$. We may choose a sequence $\left\{\lambda_{n}\right\}_{n=1}^{\infty}$ in supp $\mu_{x}$ which is discrete in $X$. Then a cluster point of $\left\{\lambda_{n}\right\}_{n=1}^{\infty}$ is continued in supp $\mu_{x}$.

6. Singly generated Douglas algebras. In this section, we answer the following problem given in $[6,19]$; when is $\left[H^{\infty}, f\right], f \in L^{\infty}$, singly generated? The characterization of singly generated Douglas algebras in [14] does not answer the above problem explicitly. We want to know conditions on $f$ satisfying that $\left[H^{\infty}, f\right]$ is singly generated.

For a point $y$ in $M(Q C)$ and $f \in L^{\infty}$, we put

$$
\left\|f+H^{\infty}\right\|_{y}=\inf _{h \in H^{\infty}}\left\{\sup |f(x)+h(x)| ; x \in \pi^{-1}(y)\right\} .
$$

By [23], the set $\left\{\left\|f+H^{\infty}\right\|_{y} ; y \in M(Q C)\right\}$ contains 0 . First we shall prove the following proposition, which is interesting in its own right. 
Proposition 6.1. For a given $f \in L^{\infty}$, the map $Q C \ni y \rightarrow\left\|f+H^{\infty}\right\|_{y}$ is upper semicontinuous.

PROOF. Let $r$ be a real number. Let $\left\{y_{\alpha}\right\}_{\alpha \in \Lambda}$ be a net in $M(Q C)$ such that

$$
\begin{aligned}
& y_{\alpha} \rightarrow y_{0} \in M(Q C), \\
& \left\|f+H^{\infty}\right\|_{y_{\alpha}} \geq r \quad \text { for every } \alpha \in \Lambda .
\end{aligned}
$$

We shall show that $\left\|f+H^{\infty}\right\|_{y_{0}} \geq r$. Since $\pi^{-1}\left(y_{\alpha}\right)$ is a weak peak set for $H^{\infty}$, by (2) there is a measure $\mu_{\alpha}$ such that

$$
\begin{gathered}
\left\|\mu_{\alpha}\right\|=1 \text { and } \operatorname{supp} \mu_{\alpha} \subset \pi^{-1}\left(y_{\alpha}\right), \\
\int_{X} f d \mu_{\alpha}=\left\|f+H^{\infty}\right\|_{y_{\alpha}} \text { and } \mu_{\alpha} \perp H^{\infty} .
\end{gathered}
$$

Let $\mu_{0}$ be a weak ${ }^{*}$-cluster point of $\left\{\mu_{\alpha}\right\}_{\alpha \in \Lambda}$, that is, $\int_{X} g d \mu_{\alpha} \rightarrow \int_{X} g d \mu_{0}$ for every $g \in C(X)$. Then $\left\|\mu_{0}\right\| \leq 1$. By (2) and (4), we have

$$
\int_{X} f d \mu_{0} \geq \inf _{\alpha} \int_{X} f d \mu_{\alpha}=\inf _{\alpha}\left\|f+H^{\infty}\right\|_{y_{\alpha}} \geq r .
$$

We note that $\pi^{-1}\left(y_{0}\right)$ is also a weak peak set for $Q A$. Let $h \in Q A$ be any peaking function such that $\pi^{-1}\left(y_{0}\right) \subset\{x \in X ; h(x)=1\}$. Since $h$ is constant on each $Q C$-level set, we have $\int_{X} f h d \mu_{\alpha}=h\left(y_{\alpha}\right) \int_{X} f d \mu_{\alpha}$ by (3). Thus

$$
\int_{X} f h d \mu_{0}=\lim _{\alpha} h\left(y_{\alpha}\right) \int_{X} f d \mu_{\alpha}=\int_{X} f d \mu_{0} \quad \text { by (1). }
$$

This shows that $\int_{\pi^{-1}\left(y_{0}\right)} f d \mu_{0}=\int_{X} f d \mu_{0} \geq r$. By $[4$, p. 58] and (4), we have $\mu_{0} \mid \pi^{-1}\left(y_{0}\right) \perp H^{\infty}$. Since $\left\|\mu_{0}\right\| \leq 1,\left\|f+H^{\infty}\right\|_{y_{0}} \geq r$. This completes the proof.

Our theorem is

THEOREM 6.1. Let $f \in L^{\infty}$. Then the following assertions are equivalent.

(i) $\left[H^{\infty}, f\right]$ is singly generated.

(ii) $Q(f)$ is a closed subset of $X$, consequently $Q(f)=N(f)$.

(iii) In the set $\left\{\left\|f+H^{\infty}\right\|_{y} ; y \in M(Q C)\right\}, 0$ is an isolated point.

PROOF. (i) $\Rightarrow$ (ii) follows from Lemma 3.1.

(ii) $\Rightarrow$ (iii). Suppose that 0 is not isolated in the set $\left\{\left\|f+H^{\infty}\right\|_{y} ; y \in M(Q C)\right\}$. Then there is a sequence $\left\{y_{n}\right\}_{n=1}^{\infty}$ in $M(Q C)$ such that $0<\left\|f+H^{\infty}\right\|_{y_{n}}<1 / n$ for $n=1,2, \ldots$. Taking a subsequence, we may assume that $\left\{y_{n}\right\}_{n=1}^{\infty}$ is discrete in $M(Q C)$. Since $0<\left\|f+H^{\infty}\right\|_{y_{n}}, \pi^{-1}\left(y_{n}\right)$ is not simple. Thus $\left\{y_{n}\right\}_{n=1}^{\infty}$ is strongly discrete. Let $h_{n} \in H^{\infty}$ with

$$
\sup \left\{\left|\left(f+h_{n}\right)(x)\right| ; x \in \pi^{-1}\left(y_{n}\right)\right\}<1 / n .
$$

Since $\pi^{-1}\left(y_{n}\right)$ is a weak peak set for $H^{\infty}$, we may assume that $\left\{h_{n}\right\}_{n=1}^{\infty}$ is a bounded sequence in $H^{\infty}$. By Theorem 5.2, there is $F \in H^{\infty}$ such that $F=h_{n}$ on $\pi^{-1}\left(y_{n}\right)$. By (1),

$$
\sup \left\{|(f+F)(x)| ; x \in \pi^{-1}\left(y_{n}\right)\right\}<1 / n .
$$

Let $y_{0} \in M(Q C)$ be a cluster point of $\left\{y_{n}\right\}_{n=1}^{\infty}$. By Theorem 5.1, $\pi^{-1}\left(y_{0}\right) \subset$ $\operatorname{cl}\left(\bigcup_{n=1}^{\infty} \pi^{-1}\left(y_{n}\right)\right) \subset N(f)$. By $(2),(f+F)(x)=0$ for $x \in \pi^{-1}\left(y_{0}\right)$. Thus $f\left|\pi^{-1}\left(y_{0}\right) \in H^{\infty}\right| \pi^{-1}\left(y_{0}\right)$, so $Q(f) \varsubsetneqq N(f)$. 
(iii) $\Rightarrow$ (i) Suppose that 0 is isolated in the set $\left\{\left\|f+H^{\infty}\right\|_{y} ; y \in M(Q C)\right\}$. Then there is $\varepsilon>0$ such that

$$
\left\{y \in M(Q C) ;\left\|f+H^{\infty}\right\|_{y} \neq 0\right\}=\left\{y \in M(Q C) ;\left\|f+H^{\infty}\right\|_{y} \geq \varepsilon\right\} .
$$

By Proposition 6.1, $\left\{y \in M(Q C) ;\left\|f+H^{\infty}\right\|_{y} \neq 0\right\}$ is a closed subset of $M(Q C)$. Hence $\pi^{-1}\left\{y \in M(Q C) ;\left\|f+H^{\infty}\right\|_{y} \neq 0\right\}=N(f)$ by Corollary 2.1. Let $I$ be an inner function such that $\bar{I} \in\left[H^{\infty}, f\right]$ and $\left\|I f+H^{\infty}\right\|<\varepsilon$. We note that if $y \in$ $M(Q C)$ satisfies $\left\|f+H^{\infty}\right\|_{y} \neq 0$, then $\pi^{-1}(y) \subset N(\bar{I})$. For, if $\pi^{-1}(y) \cap N(\bar{I})=\varnothing$ then $\varepsilon \leq\left\|f+H^{\infty}\right\|_{y}=\left\|I f+H^{\infty}\right\|_{y}<\varepsilon$. Hence $N(f) \subset N(\bar{I})$. By Corollary 2.5, we get $\left[H^{\infty}, f\right] \subset\left[H^{\infty}, \bar{I}\right] \subset\left[H^{\infty}, f\right]$.

The following corollary was proved by Marshall [19].

COROLLARY 6.1. $\left[H^{\infty}, \chi_{U}\right]$ is singly generated for every open and closed subset $U$ of $X$.

ProOF. We shall show that for $y \in M(Q C)$ either $\left\|\chi_{U}+H^{\infty}\right\|_{y}=1 / 2$ or $\left\|\chi_{U}+H^{\infty}\right\|_{y}=0$. It is easy to see that $\left\|\chi_{U}+H^{\infty}\right\|_{y} \leq 1 / 2$. Suppose $\left\|\chi_{U}+H^{\infty}\right\|_{y}<$ $1 / 2$. There is $h \in H^{\infty}$ such that $\sup _{x \in \pi^{-1}(y)}\left|\chi_{U}(x)+h(x)\right|<1 / 2$. Then there is a sequence of analytic polynomials $\left\{p_{n}\right\}_{n=1}^{\infty}$ such that $p_{n} \circ h \rightarrow \chi_{U}$ uniformly on $\pi^{-1}(y)$. Thus $\left\|\chi_{U}+H^{\infty}\right\|_{y}=0$. By Theorem 6.1, we get our assertion.

We shall give an example concerning countable valued functions.

EXAMPLE. There exist two functions $f$ and $g$ in $L^{\infty}$ such that

(a) $f(X)=g(X)=\{0,1 / n ; n=1,2, \ldots\}$,

(b) $\left[H^{\infty}, g\right]$ is not singly generated, and

(c) $\left[H^{\infty}, f\right]$ is singly generated.

PROOF. Let $\left\{O_{n}\right\}_{n=1}^{\infty}$ be a sequence of open arcs such that $O_{n}=\left\{e^{i \theta} ; 1 / n+1<\right.$ $\theta<1 / n\}$. Put $U_{n}=\left\{x \in X ; \chi_{O_{n}}(x)=1\right\}$. Then $U_{n}$ is an open and closed subset of $X$. Put

$$
g= \begin{cases}\sum_{n=1}^{\infty} \frac{1}{n} \chi_{O_{n}} & \text { on } \bigcup_{n=1}^{\infty} O_{n}, \\ 0 & \text { on } \partial D \backslash \bigcup_{n=1}^{\infty} O_{n} .\end{cases}
$$

By the same way as the proof of Corollary 6.1,

$$
\left\{\left\|g+H^{\infty}\right\|_{y} ; y \in M(Q C)\right\}=\{0,1 / 2\} \cup\{(1 / n-1 / n+1) / 2 ; n=1,2, \ldots\} .
$$

By Theorem 6.1, $g$ satisfies (a) and (b). Put

$$
f= \begin{cases}\sum_{n=1}^{\infty} \frac{1}{n} \chi_{O_{2 n}} & \text { on } \bigcup_{n=1}^{\infty} O_{2 n} \\ 1 & \text { on } \partial D \backslash \bigcup_{n=1}^{\infty} O_{2 n} .\end{cases}
$$

Then $\left\{\left\|f+H^{\infty}\right\|_{y} ; y \in M(Q C)\right\}=\{(1-1 / n) / 2 ; n=1,2, \ldots\}$. By Theorem $6.1, f$ satisfies (a) and (c).

7. $M$-ideals. Let $F$ be a weak peak subset of $X$ for $H^{\infty}+C$. We put $\left(H^{\infty}+C\right)_{F}=\left\{f \in L^{\infty} ; f\left|F \in H^{\infty}+C\right| F\right\}$. Then $\left(H^{\infty}+C\right)_{F}$ is a Douglas algebra. In [18], Luecking and Younis gave the following conjecture: Let $B$ be a Douglas algebra such that $B / H^{\infty}$ is an $M$-ideal of $L^{\infty} / H^{\infty}$. Is $B=\left(H^{\infty}+C\right)_{F}$ for some weak peak set $F$ for $H^{\infty}+C$ ? We shall give a negative answer. 
THEOREM 7.1. Let $E \varsubsetneqq X$ be a peak set for $Q C$. Put

$$
B=\left[H^{\infty},\{\bar{I} ; I \text { is an inner function with } N(\bar{I}) \subset E\}\right] \text {. }
$$

Then

(i) $B / H^{\infty}$ is an $M$-ideal of $L^{\infty} / H^{\infty}$.

(ii) $B \neq\left(H^{\infty}+C\right)_{F}$ for every weak peak set $F$ for $H^{\infty}+C$.

To show this, we need some lemmas.

Lemma $7.1[\mathbf{1 6}$, COROLlaRY 5.1]. Let $B$ be a Douglas algebra with $B \supsetneqq$ $H^{\infty}+C$. Then $B / H^{\infty}$ is an $M$-ideal of $L^{\infty} / H^{\infty}$ if and only if $B / H^{\infty}+C$ is an $M$-ideal of $L^{\infty} / H^{\infty}+C$.

The following lemma is a characterization of $M$-ideals of $L^{\infty} / H^{\infty}+C$, which is obtained by [5] essentially. For a Douglas algebra $B$, we denote by $B^{\perp}$ the space of annihilating measures on $X$ for $B$.

LEMma 7.2 (SEe [16, TheOREM 5.1]). Let $B$ be a Douglas algebra with $B \supsetneqq H^{\infty}+C$. Then $B / H^{\infty}+C$ is an $M$-ideal of $L^{\infty} / H^{\infty}+C$ if and only if for each $\mu \in\left(H^{\infty}+C\right)^{\perp}$ there exists $f_{\mu} \in L^{1}(|\mu|)$ such that

(a) $f_{\mu}^{2}=f_{\mu}$ a.e. $d|\mu|$,

(b) $\mu-f_{\mu} \mu \perp B^{\perp}$, and

(c) $f_{\mu} \mu \in B^{\perp}$.

For a subset $E$ of $X$, we put $\Lambda_{E}=\{I ; I$ is an inner function with $N(\bar{I}) \subset E\}$. As applications of Lemma 2.5 and Theorem 2.1, we get the following lemma.

LEMMA 7.3. Let $E \subset X$ be a peak set for $Q C$. For a sequence of inner functions $\left\{I_{n}\right\}_{n=1}^{\infty}$ in $\Lambda_{E}$, there exists $I \in \Lambda_{E}$ such that $N\left(\bar{I}_{n}\right) \subset N(\bar{I})$ for all $n$.

ProOF. Let $h \in Q C$ be a peaking function for $E$. By Lemma 2.1, we may assume that each $I_{n}$ is an interpolating Blaschke product with zeros $\left\{z_{n, k}\right\}_{k=1}^{\infty}$ and $\sum_{n=1}^{\infty} \sum_{k=1}^{\infty}\left(1-\left|z_{n, k}\right|\right)<\infty$. Then $\prod_{n=1}^{\infty} I_{n}$ is a Blaschke product. Put $\psi=\prod_{n=1}^{\infty} I_{n}$ and $g=\bar{\psi}(1-h)$. Then

$$
N(\bar{\psi}) \backslash E \subset N(g) .
$$

To prove that $\left\{I_{n}\right\}_{n=1}^{\infty}$ and $g$ satisfy the assumptions of Lemma 2.5, let $x \in$ $M\left(H^{\infty}+C\right)$. Since $E$ is a union set of some $Q C$-level sets, supp $\mu_{x} \subset E$ or $\operatorname{supp} \mu_{x} \cap E=\varnothing$. If $\operatorname{supp} \mu_{x} \subset E$, we get $0=g\left|\operatorname{supp} \mu_{x} \in H^{\infty}\right| \operatorname{supp} \mu_{x}$. If $\operatorname{supp} \mu_{x} \cap E=\varnothing$, then $\bar{I}_{n}\left|\operatorname{supp} \mu_{x} \in H^{\infty}\right| \operatorname{supp} \mu_{x}$ for all $n$, because $I_{n} \in \Lambda_{E}$. By Lemma 2.5 , there is a Blaschke product $I$ such that

(2) $N(\bar{I}) \subset N(\bar{\psi})$,

(3) either $\bar{I}\left|\operatorname{supp} \mu_{x} \in H^{\infty}\right| \operatorname{supp} \mu_{x}$ or $g\left|\operatorname{supp} \mu_{x} \in H^{\infty}\right| \operatorname{supp} \mu_{x}$ for every $x \in$ $M\left(H^{\infty}+C\right)$, and

(4) $N\left(\bar{I}_{n}\right) \subset N(\bar{I})$ for all $n$.

By (3), applying Theorem 2.1, we get $N(\bar{I}) \cap N(g)=\varnothing$. Hence, by (1) and (2), $N(\bar{I}) \subset E$, so $I \in \Lambda_{E}$. 
Lemma 7.4 [13, Lemma 3]. Let $E$ be a closed $G_{\delta}$-subset of $X$. Then there is an inner function $I$ with $\varphi \neq N(\bar{I}) \subset E$.

LEMMA 7.5. Let $\nu$ be a measure on $X$ with $\nu \in\left(H^{\infty}+C\right)^{\perp}$. If $J$ is an inner function with $\bar{J} \nu \notin\left(H^{\infty}+C\right)^{\perp}$, then $|\nu|(N(\bar{J})) \neq 0$.

Proof. Suppose that $|\nu|(N(\bar{J}))=0$. Then there is a sequence of compact subsets $\left\{K_{n}\right\}_{n=1}^{\infty}$ of $X$ such that $\lim _{n \rightarrow \infty}|\nu|\left(K_{n}\right)=\|\nu\|$ and $K_{n} \cap N(\bar{J})=\varnothing$. Since $\pi^{-1}(\pi(N(\bar{J})))=N(\bar{J})$, moreover we may assume $\pi^{-1}\left(\pi\left(K_{n}\right)\right)=K_{n}$. Then $K_{n}$ is a weak peak set for $Q C$ and for $H^{\infty}+C$. Hence $\nu \mid K_{n} \in\left(H^{\infty}+C\right)^{\perp}[\mathbf{4}$, p. 58]. Since $K_{n} \cap N(\bar{J})=\varnothing, \bar{J}\left|K_{n} \in\left(H^{\infty}+C\right)\right| K_{n}$. Hence $\bar{J} \nu \mid K_{n} \in\left(H^{\infty}+C\right)^{\perp}$. Since $\lim _{n \rightarrow \infty}|\nu|\left(K_{n}\right)=\|\nu\|, \bar{J} \nu \in\left(H^{\infty}+C\right)^{\perp}$. But this is a contradiction.

LEMMA 7.6 [16, THEOREM 2.1]. Let $B$ be a Douglas algebra with $B \supset$ $H^{\infty}+C$. Let $\lambda$ be a measure on $X$ with $\lambda \in B^{\perp}$. If $\nu$ is a measure with $\nu \ll \lambda$, then there is an inner function $I$ such that $I \nu \in B^{\perp}$.

Proof of TheOREM 7.1. (i) By Lemma 7.4, $H^{\infty}+C \varsubsetneqq B$. We shall show that $B / H^{\infty}+C$ is an $M$-ideal of $L^{\infty} / H^{\infty}+C$, then we get (i) by Lemma 7.1. To show the above fact, we use Lemma 7.2. Let $\mu \in\left(H^{\infty}+C\right)^{\perp}$ with $\|\mu\|=1$. Put $\alpha=\sup \left\{|\mu|(N(\bar{I})) ; I \in \Lambda_{E}\right\}$. Then there is a sequence $\left\{I_{n}\right\}_{n=1}^{\infty}$ in $\Lambda_{E}$ such that $\lim _{n \rightarrow \infty}|\mu|\left(N\left(\bar{I}_{n}\right)\right)=\alpha$. By Lemma 7.3, there is $I_{0} \in \Lambda_{E}$ such that $N\left(\bar{I}_{n}\right) \subset N\left(\bar{I}_{0}\right)$. Hence $|\mu|\left(N\left(\bar{I}_{0}\right)\right)=\alpha$. Put $f_{\mu}=1-\chi_{N\left(\bar{I}_{0}\right)}$. Then $f_{\mu}$ satisfies (a) of Lemma 7.2. Also by Lemma 7.3,

$$
\left|f_{\mu} \mu\right|(N(\bar{I}))=0 \quad \text { for every } I \in \lambda_{E} .
$$

To show $f_{\mu} \mu \in B^{\perp}$, suppose that $f_{\mu} \mu \notin B^{\perp}$. Since $B$ coincides with the closed linear span of $\left\{\bar{I}\left(H^{\infty}+C\right) ; I \in \Lambda_{E}\right\}$, there is $J \in \Lambda_{E}$ such that $\bar{J} f_{\mu} \mu \notin$ $\left(H^{\infty}+C\right)^{\perp}$. We note that $f_{\mu} \mu \in\left(H^{\infty}+C\right)^{\perp}$, because $N\left(\bar{I}_{0}\right)$ is a weak peak set for $H^{\infty}+C$ by Corollary 2.1. By Lemma 7.5, $\left|f_{\mu} \mu\right|(N(\bar{J})) \neq 0$. But this contradicts (1). Thus we get (c) of Lemma 7.2.

To prove (b), we shall show

(2) $\lambda \mid N(\bar{I})=0$ for every $\lambda \in B^{\perp}$ and $I \in \Lambda_{E}$.

Fix $\lambda \in B^{\perp}$ and $I \in \Lambda_{E}$. By Lemma 7.6, there is an inner function $\Psi$ such that

$$
\Psi|\lambda| \mid N(\bar{I}) \in B^{\perp} .
$$

Let $h \in Q C$ be a peaking function for $E$. We note that for $x \in M\left(H^{\infty}+C\right)$, either $\bar{I}\left|\operatorname{supp} \mu_{x} \in H^{\infty}\right| \operatorname{supp} \mu_{x}$ or $\bar{\Psi}(1-h)\left|\sup \mu_{x} \in H^{\infty}\right| \operatorname{supp} \mu_{x}$, because $N(\bar{I}) \subset E$. By Theorem 2.1, $N(\bar{I}) \cap N(\bar{\Psi}(1-h))=\varnothing$. By Corollary 2.1, there is a function $q$ in $Q C$ such that $0 \leq q \leq 1$,

$$
q=1 \quad \text { on } N(\bar{I}) \quad \text { and } \quad q=0 \quad \text { on } N(\bar{\Psi}(1-h)) .
$$

If $q \bar{\Psi}\left|\operatorname{supp} \mu_{x} \notin H^{\infty}\right| \operatorname{supp} \mu_{x}$ for $x \in M\left(H^{\infty}+C\right)$, then $q(x) \neq 0$ and $\bar{\Psi} \mid \operatorname{supp} \mu_{x} \notin$ $H^{\infty} \mid \operatorname{supp} \mu_{x}$. Since $h \in Q C, h(x)=1$ by (4). Hence $\operatorname{supp} \mu_{x} \subset E$, so $N(q \bar{\Psi}) \subset$ $E$. By Lemma 2.2, there is a sequence of inner functions $\left\{\Psi_{n}\right\}_{n=1}^{\infty}$ such that $\left[H^{\infty}, q \bar{\Psi}\right]=\left[H^{\infty},\left\{\bar{\Psi}_{n}\right\}_{n=1}^{\infty}\right]$. Since $N\left(\bar{\Psi}_{n}\right) \subset N(q \bar{\Psi}) \subset E$, we get $\left[H^{\infty}, q \bar{\Psi}\right] \subset B$. By (3) and (4),

$$
0=\int_{N(\bar{I})} q \bar{\Psi} \Psi d|\lambda|=\int_{N(\bar{I})} d|\lambda| .
$$


Hence $\lambda \mid N(\bar{I})=0$. Thus we get (2). Consequently $\mu-f_{\mu} \mu=\mu \mid N\left(\bar{I}_{0}\right) \perp B^{\perp}$, so we get (b) of Lemma 7.2.

Applying Lemma 7.2, $B / H^{\infty}+C$ is an $M$-ideal of $L^{\infty} / H^{\infty}+C$. This completes the proof of (i).

(ii) Suppose that $B=\left(H^{\infty}+C\right)_{F}$ for a weak peak subset of $X$ for $H^{\infty}+C$. To show $F \supset X \backslash E$, suppose not. Then there exists an open and closed subset $U$ of $X$ with $U \cap(E \cup F)=\varnothing$. By Lemma 7.4, there is an inner function $I$ with $\varnothing \neq N(\bar{I}) \subset U$. Then there is $x \in M\left(H^{\infty}+C\right) \backslash X$ such that supp $\mu_{x} \subset U$. Since $\operatorname{supp} \mu_{x} \cap E=\varnothing, B\left|\operatorname{supp} \mu_{x}=H^{\infty}\right| \operatorname{supp} \mu_{x}$. Since $\operatorname{supp} \mu_{x} \cap F=\varnothing$, $\left(H^{\infty}+C\right)_{F} \mid \operatorname{supp} \mu_{x}$ coincides with the space of continuous functions on supp $\mu_{x}$. This is a contradiction, so we have $F \supset X \backslash E$.

Let $V$ be the closure of $X \backslash E$. By [4, p. 18], $V$ is an open and closed subset of $X$ and $V \subset F$. Since $Q C$ does not have nontrivial idempotents, $E \cap V \neq \varnothing$. Since $E \cap V$ is a closed $G_{\delta}$-set, again by Lemma 7.4 there is an inner function $J$ with $\varnothing \neq N(\bar{J}) \subset E \cap V$. By the definition of $B, \bar{J} \in B$. Since $N(\bar{J}) \subset V \subset F, \bar{J} \notin$ $\left(H^{\infty}+C\right)_{F}$. This contradicts $B=\left(H^{\infty}+C\right)_{F}$. Hence we get (ii).

REMARK. Let $B$ be a Douglas algebra given in Theorem 7.1. By the above proof and [4, p. 59], $N(\bar{I})$ is an interpolation set for $B$ for every $I \in \Lambda_{E}$, that is, $B \mid N(\bar{I})=C(N(\bar{I}))$. If we put $B^{\prime}=\left[H^{\infty},\{\bar{I}: I\right.$ is an inner function with $N(\bar{I}) \subset$ $X \backslash E\}]$, then we have $B^{\prime}=\left(H^{\infty}+C\right)_{E}$

By the same way as in $[\mathbf{1 7}]$, we have the following.

COROLlary 7.1. Let $B$ be a Douglas algebra given in Theorem 7.1. Then $B$ has the best approximation property, that is, for each $f \in L^{\infty}$ there is $g \in B$ such that $\|f+B\|=\|f-g\|$.

As a special case, we get Proposition 2 in $[\mathbf{1 8}]$. Let $F$ be an open subset of $\partial D$. Put $L_{F}^{\infty}=\left\{f \in L^{\infty} ; f\right.$ is continuous at each point of $\left.F\right\}$, and $E=\{x \in X ; z(x) \in$ $X \backslash F\}$. Then $E$ is a peak set for $Q C$, and it is easy to see that $H^{\infty}+L_{F}^{\infty}=$ $\left[H^{\infty},\{\bar{I} ; I\right.$ is an inner function with $\left.N(\bar{I}) \subset E\}\right]$. Hence $H^{\infty}+L_{F}^{\infty}$ has the best approximation property.

\section{REFERENCES}

1. S. Axler, Factorization of $L^{\infty}$ functions, Ann. of Math. (2) 106 (1977), 567-572.

2. S. Axler, I. D. Berg, N. Jewell, and A. L. Shields, Approximation by compact operators and the space $H^{\infty}+C$, Ann. of Math. (2) 109 (1979), 601-612.

3. S.-Y. A. Chang, A characterization of Douglas subalgebras, Acta Math. 137 (1976), 81-89.

4. T. Gamelin, Uniform algebras, Prentice-Hall, Englewood Cliffs, N.J., 1969.

5. T. Gamelin, D. Marshall, R. Younis, and W. Zame, Function theory and M-ideals, Ark. Mat. 23 (1985), 261-279.

6. J. Garnett, Bounded analytic functions, Academic Press, New York and London, 1981.

7. P. Gorkin, Decompositions of the maximal ideal space of $L^{\infty}$, Thesis, Michigan State Univ., East Lansing, 1982.

8. ___ Decompositions of the maximal ideal space of $L^{\infty}$, Trans. Amer. Math. Soc. 282 (1984), 33-44.

9. C. Guillory, K. Izuchi and D. Sarason, Interpolating Blaschke products and division in Douglas algebras, Proc. Roy. Irish Acad. 84A (1984), 1-7.

10. K. Hoffman, Banach spaces of analytic functions, Prentice-Hall, Englewood Cliffs, N.J., 1962.

11. $\ldots$, unpublished note. 
12. K. Izuchi, Zero sets of interpolating Blaschke products, Pacific J. Math. 119 (1985), 337 342 .

13. __ Q Q - Q level sets and quotients of Douglas algebras, J. Funct. Anal. 65 (1986), 293-308.

14. __ A geometrical characterization of singly generated Douglas algebras, Proc. Amer. Math. Soc. 97 (1986), 410-412.

15. K. Izuchi and Y. Izuchi, Extreme and exposed points in quotients of Douglas algebras by $H^{\infty}$ or $H^{\infty}+C$, Yokohama Math. J. 32 (1984), 45-54.

16. __ Annihilating measures for Douglas algebras, Yokohama Math. J. 32 (1984), 135-151.

17. D. Luecking, The compact Hankel operators from an $M$-ideal in the space of Hankel operators, Proc. Amer. Math. Soc. 79 (1980), 222-224.

18. D. Luecking and R. Younis, Quotients of $L^{\infty}$ by Douglas algebras and best approximation, Trans. Amer. Math. Soc. 276 (1983), 699-706.

19. D. Marshall, Subalgebras of $L^{\infty}$ containing $H^{\infty}$, Acta Math. 137 (1976), 91-98.

20. D. Sarason, Functions of vanishing mean oscillation, Trans. Amer. Math. Soc. 207 (1975) 391-405.

21. Function theory on the unit circle, Virginia Polytechnic Institute and State Univ., Blacksburg, 1978.

22. __ The Shilov and Bishop decompositions of $H^{\infty}+C$, Conf. on Harmonic Anal. in Honor of A. Zygmund, Vol. 2, Wadsworth, Belmont, Calif., 1981, pp. 461-474.

23. T. Wolff, Two algebras of bounded functions, Duke Math. J. 49 (1982), 321-328.

24. R. Younis, Division in Douglas algebras and some applications, Arch. Math. (Basel) 45 (1985), 550-560.

Department of Mathematics, Kanagawa University, Yokohama 221, Japan 